\title{
Big Observation - Ein Vergleich moderner Beobachtungsformate am Beispiel von amtlicher Statistik und Recommendersystemen
}

\author{
Bettina Heintz
}

Eingegangen: 4. Januar 2021 / Angenommen: 17. Januar 2021 / Online publiziert: 4. Mai 2021

(C) Der/die Autor(en) 2021

Zusammenfassung Der Beitrag verortet digitale Technologien in der Geschichte moderner Beobachtungsformate. Das Konzept des Beobachtungsformats verhilft dazu, Einrichtungen in einen Zusammenhang zu bringen, die auf den ersten Blick wenig miteinander zu tun haben: die amtliche Statistik, die Meinungs- und Marktforschung, Monitoringsysteme und digitale Beobachtungsinstrumente wie Suchmaschinen, soziale Netzwerke oder Recommendersysteme. Die Gemeinsamkeit liegt darin, dass es sich in allen Fällen um Instanzen handelt, die regelmäßig Daten erheben und in diesen Daten nach Ordnungsmustern suchen. Sie sind markante Beispiele des Quantifizierungsschubs, der die (westlichen) Gesellschaften seit dem 18. Jahrhundert erfasst hat. Der Beitrag greift zwei Beispiele heraus - die Bevölkerungsstatistik als historisch erstes modernes Beobachtungsformat und personalisierte Recommendersysteme als prototypischen Fall digitaler Beobachtungsinstrumente und vergleicht sie in Hinblick auf ihre Beobachtungstechnik: Wie werden die Zahlen fabriziert, mit deren Hilfe die amtliche Statistik die Gesellschaft beobachtet, und welche Beobachtungsverfahren setzen Recommendersysteme ein, um zu personalisierten Empfehlungen zu gelangen, und welche Rolle spielen dabei Vergleich, Bewertung, Kategorisierung und Quantifizierung? Der Vergleich macht nicht nur sichtbar, wie Statistiken und digitale Technologien beobachten und worin sich ihre Beobachtungstechnik unterscheidet, sondern er gibt auch Aufschluss darüber, wie sich die Praktiken und Prämissen sozialer Beobachtung in den letzten 200 Jahren verändert haben.

Schlüsselwörter Geschichte der Statistik · Digitalisierung · Soziale Vergleiche · Quantifizierung · Kategorisierung

B. Heintz $(\bowtie)$

Soziologisches Seminar, Universität Luzern

Frohburgstr. 3, 6002 Luzern, Schweiz

E-Mail: bettina.heintz@unilu.ch 


\title{
Big Observation-Comparing Population Statistics and Recommender Systems as Modern Formats of Observation
}

\begin{abstract}
The article situates digital technologies in the history of modern formats of observation. The term "format of observation" helps to relate devices and systems that at first sight do not have much in common: official statistics, opinion and market research, monitoring instruments, and digital technologies such as search engines, social networks, and recommender systems. Despite their differences, they share one important characteristic: By collecting huge amounts of data and by systematically looking for patterns in these data, they are striking examples of the rise of quantification since the 18th century. This article draws on two examples-population statistics as the historically first modern format of observation and recommender systems as a prominent example of digital observation technologies — and compares them with regard to their technique of observation. How do statisticians produce the numbers that are represented in statistical tables? And how do recommender systems calculate personalized recommendations? Comparing these two cases not only sheds light on how official statistics and digital technologies observe but also provides insight into the practices and premises of social observation over the last two hundred years.
\end{abstract}

Keywords History of statistics · Digitization - Social comparisons · Quantification · Classification

\section{Einleitung}

Die Geschichte moderner Beobachtungsformate beginnt im letzten Drittel des 18. Jahrhunderts mit der Entstehung der Statistik. Während sich die früheren Erhebungen darauf beschränkten, zu zählen und diese Zahlen lokal zu Kontrollzwecken und zur Akquirierung von Ressourcen einsetzten, ging es nun darum, durch rechnerische Operationen nach den sozialen Gesetzmäßigkeiten zu suchen, die sich hinter dem ,Rauschen der individuellen Handlungen“ (Köhler 2008, S. 76) verbergen. Das entscheidend Neue lag nicht darin, dass immer mehr Daten produziert wurden Big Data in ihren Anfängen -, neu war vielmehr die Auffassung, dass man im Wust der Daten Regelmäßigkeiten entdecken und aus diesen Planungs- und Regulierungswissen ableiten konnte (Behrisch 2016). Erst die moderne Statistik produzierte jene Makrogrößen, die für uns heute so evident sind: Bevölkerungswachstum, Nationaleinkommen, Arbeitslosenrate, Kriminalitätsrate und viele andere mehr, und erst sie machte die Zusammenhänge sichtbar, an denen sich die „Gesetze der Gesellschaft“ (Hacking 1994) erkennen ließen. Zusammengenommen sind es vor allem drei Merkmale, die die moderne Statistik von ihren Vorläufern unterscheiden und sie zum ersten modernen gesellschaftlichen Beobachtungsformat machen: das Interesse an der Entdeckung von übergreifenden Zusammenhängen, die Fokussierung auf das Vergleichen in zeitlicher, räumlicher und sozialer Hinsicht und die regelmäßige Wiederholung der Datenerhebungen, die die Feststellung von Fort- und Rückschritten überhaupt erst möglich machte. 
Über das Konzept des Beobachtungsformats lassen sich Einrichtungen in einen Zusammenhang bringen, die auf den ersten Blick wenig miteinander zu tun haben: die amtliche Statistik, die die Gesellschaft für die politischen Instanzen „lesbar“ macht (Scott 1999), die Meinungsforschung, die über die Vermessung von Einstellungen und Werten das Innere des Averaged Man ergründen will (Igo 2007), die Marktforschung, die die Präferenzen und Konsumgewohnheiten der Kunden zu ermitteln versucht (Arvidsson 2004; Turow 1997), staatlich implementierte Monitoringsysteme, die langfristige Entwicklungen anhand quantitativer Kenngrößen beobachten und daraus Handlungsempfehlungen ableiten (Petzke 2021 in diesem Band), und schließlich digitale Beobachtungsinstrumente, die das, was wir tun, in Echtzeit beobachten und dieses Tun nach Regelmäßigkeiten durchforsten.

Diese Beispiele unterscheiden sich in mancher Hinsicht. Im einen Fall ist der Staat die Beobachtungsinstanz, im anderen Fall sind es private Unternehmen, die Individuen nicht mehr in ihrer Rolle als Bürger, sondern als Konsumenten beobachten. Bei den einen liegt der Fokus auf den statistischen Aggregaten und den in ihnen verborgenen sozialen Gesetzmäßigkeiten, die anderen sammeln und analysieren Daten, um aus ihnen etwas über das zukünftige Verhalten des Einzelnen in Erfahrung zu bringen. ${ }^{1}$ Aber trotz dieser Unterschiede teilen sie eine entscheidende Gemeinsamkeit: Es handelt sich in allen Fällen um Beobachtungsformate, die regelmäßig Zahlen produzieren und in diesen Zahlen nach Mustern suchen, um auf diese Weise systematische Erkenntnisse über das Verhalten der Individuen und die Beschaffenheit der Gesellschaft zu gewinnen. Sie sind insofern Teil des Quantifizierungsschubs, der die (westlichen) Gesellschaften seit dem 18. Jahrhundert erfasst hat (Porter 1995).

Die vorläufig letzte Entwicklung in der Geschichte moderner Beobachtungsformate sind digitale Beobachtungstechnologien, die ständig und überall im Einsatz sind und praktisch nichts mehr unbeleuchtet lassen - Shoppingportale und Streamingdienste, Suchmaschinen, soziale Netzwerke, Selbstvermessungsgeräte und Vermittlungsplattformen wie Airbnb, Ebay oder Uber. Im Unterschied zur amtlichen Statistik, bei der der Staat die Beobachtungsinstanz ist und die Beobachtung an den nationalen Grenzen haltmacht, sind es bei digitalen Technologien vor allem Privatunternehmen, die die Konsumentinnen und Konsumenten rund um die Uhr und wo auch immer sie sich aufhalten beobachten. ${ }^{2}$ Wer Google, Amazon, Netflix oder auch bloß Kreditkarten nutzt, hinterlässt Datenspuren, die automatisch aufgezeichnet und auf ihre Muster hin ausgewertet werden, unabhängig davon, wo sich jemand aufhält. Mit ihrer Fähigkeit, jeden Click und jede Bewegung, jedes Hörverhalten und jede Online-Transaktion in Daten zu verwandeln und diese in Sekundenschnelle zu analysieren, transformieren diese Applikationen die Technik sozialer Beobachtung und heben sie auf ein neues Niveau.

\footnotetext{
${ }^{1}$ Zur Spannung zwischen diesen beiden Zugangsweisen siehe Bouk (2015) am Beispiel des frühen Versicherungswesens.

${ }^{2}$ Digitale Beobachtungstechnologien werden auch von staatlichen Behörden eingesetzt, aber nicht in gleichem Umfang, man denke etwa an den Einsatz von prädiktiven Algorithmen bei der Strafzumessung, der polizeilichen Ermittlung (Brayne 2021 in diesem Band) oder in der öffentlichen Verwaltung (Büchner und Dosdall 2021 in diesem Band).
} 
Im Gegensatz zu den Surveillance-Studies, die Beobachtung vor allem unter dem Aspekt der Überwachung untersuchen und digitale Technologien in der Geschichte panoptischer Kontrolle situieren (exemplarisch Haggerty und Ericson 2000; DegliEspositi 2014), soll im Folgenden das Beobachten selbst in den Mittelpunkt gerückt werden, unabhängig davon, ob die gewonnenen Informationen zu Überwachungsund Kontrollzwecken eingesetzt werden oder nicht. Digitale Beobachtungstechnologien verarbeiten zwar Unmengen von Daten - Big Data -, entscheidend ist aber weniger der Umfang an Daten, als vielmehr das, was sich mithilfe von Algorithmen in diesen Daten beobachten lässt (Amoore und Piotukh 2015). Anstatt von Big Data ließe sich deshalb ebenso gut und vielleicht sogar noch treffender von Big Observation sprechen. So gesehen hat der „known citizen“, von dem Sarah Igo (2018) in ihrer Geschichte der Semantik der Privatheit spricht, erst mit den neuen Technologien wirklich Gestalt angenommen. Vorher blieb das Wissen über ihn fragmentarisch und erfasste ihn nur als gemittelten Bürger oder als Teil eines soziodemografischen Kollektivs. Dagegen zielen die Unternehmen, die heute Daten sammeln, analysieren, zusammenführen und verkaufen, darauf, Wissen über den Einzelnen anzusammeln.

Der Beitrag greift zwei Beispiele heraus - die Bevölkerungsstatistik als historisch erstes modernes Beobachtungsformat und personalisierte Recommendersyste$m e$ als prototypischen Fall digitaler Beobachtungsinstrumente - und vergleicht sie hinsichtlich der Frage, wie sie Daten erheben und in diesen Daten zur Entdeckung von Regelmäßigkeiten gelangen. Setzt man die beiden Formate in Beziehung zur Gesellschaft, in der sie entstanden sind, macht der Vergleich nicht nur sichtbar, wie die Bevölkerungsstatistik und Recommendersysteme beobachten und worin sich ihre Beobachtungstechnik unterscheidet, sondern er gibt auch Aufschluss darüber, wie sich die Prämissen sozialer Beobachtung in den letzten 200 Jahren verändert haben.

Die Verortung digitaler Beobachtungstechnologien in einer Geschichte gesellschaftlicher Beobachtungsformate ist durch die Annahme motiviert, dass die neuen Technologien nicht so umstürzend neu sind wie oft behauptet und die üblichen Umschreibungen - digital, algorithmisch, Big Data - wenig dazu taugen, ihre Andersartigkeit soziologisch auf den Begriff zu bringen. Denn die „,neuen“ Technologien sind nicht neu, weil sie „digital“ sind und Algorithmen einsetzen. „Digital“" waren schon die ersten Computer der 1940er-Jahre, die damals noch explizit Digitalcomputer genannt wurden. Ähnlich ist auch Algorithmisierung kein brandneues Phänomen, sondern steht in der Tradition des Rationalisierungsdiskurses im ersten Drittel des 20. Jahrhunderts. Auch der Kohlenschaufler, der kleinste Körperbewegungen nach Frederick W. Taylors ,,bester Methode“ auszuführen hatte, folgte einem Algorithmus, auch wenn dieser technisch noch nicht implementiert war (zu beidem ausführlicher Heintz 1993). Und ebenso ist die ständige Produktion von immer mehr Daten nicht so neu, wie sie erscheint (Ambrose 2015; Beer 2016). Schon mit dem Aufbau der amtlichen Statistik kam es zu einem rasanten Anstieg von Daten, den Ian Hacking (1982) auf die bekannte Formel einer ,avalanche of printed numbers“ brachte und Theodore Porter (1986, S. 11) als ,the great explosion of numbers“ bezeichnete.

Aber wenn Begriffe wie „Big Data“ und „Algorithmus“ nicht ausreichen, um die Neuheit digitaler Beobachtungsformate zu erfassen, wie lässt sie sich dann begreifen? Ich werde im Folgenden argumentieren, dass die Besonderheit digitaler Beobachtungstechnologien in ihrer spezifischen Operationsweise liegt und nicht bloß 
darin, dass sie Algorithmen einsetzen und auf Massen von Daten zurückgreifen. Das tun sie zwar auch, aber damit ist noch nichts darüber ausgesagt, wie sie das tun. Der Beitrag zeigt auf, dass ihr operatives Grundprinzip in der „Selbsterzeugung“ von Daten und darin besteht, dass sie Vergleich, Bewertung und Kategorisierung in einem ständigen rekursiven Prozess verknüpfen und sich diese Verknüpfung im Medium algorithmischer Berechnungen vollzieht. Wie dies genau geschieht, beschreibe ich in einem ersten Abschnitt am Beispiel von Recommendersystemen, die auf dem Verfahren des Collaborative Filtering beruhen. In der wissenschaftlichen und populären Literatur wird die Besonderheit digitaler Technologien oft daran abgelesen, wie sie sich auf der Bildschirmoberfläche präsentieren. Um ihre Eigenheit $\mathrm{zu}$ verstehen, muss man sich jedoch mit der algorithmischen Hinterbühne befassen und wird dann z. B. erkennen, dass die ihnen häufig attestierte Singularisierung und Personalisierung durch Standardisierung, Entpersonalisierung und den Vergleich mit Anderen zustande kommt: „Ich bin die Anderen“ und „,Du“ bin nicht ,ich““.

Der anschließende Abschnitt vergleicht die Operationsweise von Recommendersystemen mit der Bevölkerungsstatistik als dem exemplarischen Fall eines nichtdigitalen Beobachtungsinstruments. „Nichtdigital“ ist die amtliche Statistik insofern, als sie zwar digitalisierte Daten verwendet, aber nicht digitale Daten im strengen Sinne, also nicht Daten, die direkt aus den Netzwerkaktivitäten der Nutzerinnen und Nutzer gewonnen werden. Die Vergleichsgesichtspunkte ergeben sich aus der oben genannten Annahme, dass die Besonderheit digitaler Beobachtungstechnologien in der Art ihrer Datenerhebung und der ständigen Verknüpfung von Vergleich, Bewertung und Kategorisierung liegt. Entsprechend stellt sich auch für die Statistik die Frage, wie sie die Zahlen herstellt, mit deren Hilfe sie die Gesellschaft beobachtet, und welche Rolle die genannten Operationen dabei spielen. Während die meisten Studien zur amtlichen Statistik erst auf der Ebene der Tabellen ansetzen, rücke ich das doing statistics in den Vordergrund, d.h. die praktischen Verfahren der Fabrikation statistischer Zahlen. Denn nur auf der Ebene ihrer konkreten Operationsweise lassen sich die Mustererkennungstechniken digitaler und statistischer Beobachtungsformate sinnvoll miteinander vergleichen.

Alle Beobachtungsformate beruhen auf impliziten Theorien des Sozialen, d.h. auf Vorstellungen darüber, was sich zu zählen und $\mathrm{zu}$ beobachten lohnt und wie das Soziale, das sie zu erfassen suchen, beschaffen und strukturiert ist. Diese impliziten Theorien reflektieren das Selbstverständnis und die Problemstellungen der Gesellschaft, in der sie entstanden sind. Verortet man die Bevölkerungsstatistik und Recommendersysteme auf einer historischen Zeitachse und begreift sie als Ausdruck der Gesellschaft, in der sie entwickelt wurden, so könnten die Unterschiede in ihren Beobachtungstechniken auf einen Wandel des ihnen zugrundeliegenden Gesellschaftsverständnisses verweisen. Darauf gehe ich in einem abschließenden Teil ein. Ich schließe dabei kritisch an die Theorien des Digitalen an, die Armin Nassehi (2019) und Dirk Baecker (2018) vorgelegt haben. Mein Beitrag verfolgt zwar eine ähnliche Intention, nämlich zu untersuchen, auf welche Art von Gesellschaft digitale Technologien verweisen. Mein Zugang ist jedoch ein anderer, indem ich das, was sie auf einer allgemeinen Ebene abhandeln, anhand von zwei konkreten Fällen analysiere und an diesen beiden Fällen zeige, wie die amtliche Statistik und digitale Technologien beobachten und worin genau ihre Gemeinsamkeiten und Unterschie- 
de liegen. Durch ein solches Herunterbuchstabieren auf den konkreten Fall gewinnt man Einsichten, die einem entgehen, wenn man den Phänomenen, um die es geht, zu wenig Beachtung schenkt.

\section{Big Observation - die Beobachtungstechniken digitaler Beobachtungsformate ${ }^{3}$}

Recommendersysteme werden von praktisch allen bekannten Diensten eingesetzt: von Suchmaschinen, sozialen Netzwerken, Shoppingportalen, Streamingdiensten und Dating-Apps. Sie gehen mit dem Versprechen einher, durch algorithmisch berechnete Empfehlungen den Nutzern und Nutzerinnen im Labyrinth der Waren und Angebote einen Weg zu weisen. Wie sie das tun, unterscheidet sich jedoch. Ich beziehe mich im Folgenden auf Recommendersysteme, die auf dem Verfahren des Collaborative Filtering beruhen, da sich an ihnen die Operationsweise digitaler Beobachtungsformate besonders gut illustrieren lässt (zu dieser und anderen Varianten von Recommendersystemen Unternährer 2021a in diesem Band).

Dass Konsumentinnen und Konsumenten mit Empfehlungen versorgt werden, ist nicht neu. Das tun auch Literaturkritiken, Warentests und klassische Rankings. Neu ist aber, dass diese Empfehlungen personalisiert, d.h. auf die einzelnen Nutzer persönlich zugeschnitten sind. Wie Recommendersysteme zu solchen personalisierten Empfehlungen gelangen, lässt sich an einem vereinfachten Beispiel illustrieren. Amazon empfiehlt mir regelmäßig, welche Bücher ich lesen soll. Manchmal sind die Empfehlungen passend, manchmal aber auch nicht. Wenn Amazon registriert, dass ich mich plötzlich für ganz andere Genres interessiere - z. B. für Hunderatgeber und maschinelles Lernen - gerät das System ins Stottern. Neben Hunderatgebern und Büchern zu Deep Learning werden mir nun auch Selbsthilfebücher und FantasyRomane empfohlen. Wie kann das geschehen?

Amazon ist ein Online-Marktplatz mit eingebauter Recommenderfunktion. Wenn ich Amazon nutze, stehen mir nur wenige Verhaltensoptionen zur Verfügung - etwa suchen, anklicken, bewerten, markieren oder kaufen. Diese Aktivitäten werden von Amazon zusammen mit anderen Datenspuren aufgezeichnet, die ich hinterlasse, oft ohne es zu merken. Solche Daten hat Amazon von mehreren Millionen Kundinnen und Kunden und verfügt damit über eine gigantische Datenbasis, um mein Suchund Kaufverhalten mit jenem anderer zu vergleichen und aus diesem Vergleich Empfehlungen abzuleiten. So könnte etwa die Empfehlungsliste von Amazon auf einem Vergleich meines Interesses für Hunderatgeber und maschinellem Lernen mit dem Verhalten anderer Kunden beruhen, die ein ähnliches, aber nicht identisches Interessenprofil haben. In Amazon-Sprache: „Kunden, die diesen Artikel gekauft haben, kauften auch ...". Je besser die empfohlenen Produkte mit meinen Interessen übereinstimmen, desto eher werde ich sie auch kaufen und weiterhin bei Amazon browsen. Recommendersysteme dirigieren mit anderen Worten meine Entscheidungen in ei-

\footnotetext{
3 Ich möchte mich an dieser Stelle bei Markus Unternährer bedanken, der mich in dieses Thema eingeführt hat und dessen Texte mir geholfen haben, mich in der komplizierten Welt der digitalen Technologien zurechtzufinden (Unternährer 2021b).
} 
ne bestimmte Richtung, ohne dass ich mir der „sanften Gewalt“ der Algorithmen bewusst sein muss (Yeung 2016).

\section{1 „Selbsterzeugung“ von Daten: Standardisierung und Datafizierung}

Personalisierung setzt paradoxerweise ihr Gegenteil voraus, nämlich Standardisierung. Denn personalisierte Empfehlungen sind nur deshalb möglich, weil die Verhaltensmöglichkeiten der Nutzer auf einige wenige standardisierte Aktivitäten reduziert sind - etwa liken, teilen, folgen, markieren, kaufen. Das heißt Recommendersysteme vermessen nicht präexistente Aktivitäten, sondern sie geben hoch artifizielle Handlungsoptionen vor, die sich direkt „,datafizieren“, d.h. bei ihrer Ausführung in computerlesbare Daten transformieren lassen. Über diese Standardisierung generieren Recommendersysteme selbst die Datenobjekte, die sie anschließend analysieren (Alaimo und Kallinikos 2017; Paßmann und Gerlitz 2014). Das ist mit „Selbsterzeugung" gemeint. In gewisser Hinsicht ist der digitale Nutzer in seinen Handlungsmöglichkeiten ähnlich eingeschränkt wie der taylorisierte Arbeiter, der für Max Weber das Musterbeispiel rationaler Disziplinierung war. „Liken“, „,sharen“ oder „klicken“ sind keine „,natürlichen“ Verhaltensweisen, sondern sie sind ebenso rudimentär und artifiziell, wie die Elementaroperationen, in die Frederick W. Taylor natürliche Bewegungsabläufe zerlegt hatte. ${ }^{4}$

Im Gegensatz zu konventionellen Formen der Programmentwicklung, bei denen „natürliche“ Aktivitäten formalisiert werden müssen, um sie computertauglich zu machen, sind die zulässigen Aktivitäten bereits in so hohem Maße standardisiert, dass das Anklicken eines Share- oder Kauf-Buttons direkt vom System verarbeitbar ist. Als man in den 1950er-Jahren die ersten Computer installierte, wurden sie vor allem dort eingesetzt, wo die Arbeitsabläufe schon weitgehend standardisiert waren: in der Lohnbuchhaltung und im Rechnungswesen, kurz dort, wo die Tätigkeit schon in Einzeloperationen zerlegt war und es einen Algorithmus - eine ,,beste Methode“ - gab, wie sie auszuführen und aneinanderzureihen sind (Heintz 1990). Man habe vor allem jene „Routinearbeiten“ auf „Elektronengehirne“ umgestellt, schrieb etwa Hans-Paul Bahrdt in seiner Angestelltenuntersuchung von 1958, ,die ihre heutige Gestalt den Rationalisierungsbestrebungen der 20er-Jahre verdankten“ (Bahrdt 1958, S. 72). Um den Computer breiter einzusetzen, begann man wenig später auch Tätigkeiten zu standardisieren, die noch nicht durchgehend rationalisiert waren. Aber auch hier erforderte die Programmierung eine vorgängige Reorganisation bestehender Aktivitäten in ein formalisierbares Format.

Die Sequenz „Standardisierung - Formalisierung - Programmierung“ wird bei den neuen Technologien auf den Kopf gestellt, indem das System selbst die zulässigen Aktivitäten vorgibt. Alaimo und Kallinikos (2017) sprechen in diesem Zusammenhang von ,encoding“. ,Encoding ... does not record prior facts, which it then

\footnotetext{
4 Philip E. Agre (1994) hat solche Ketten von Anweisungen als ,grammars of action“ bezeichnet und damit auf die Kontinuitätslinie hingewiesen, die vom taylorisierten Arbeiter zu den heutigen Digitaltechnologien führt. Denn nicht nur Taylors „beste Methode“ spezifiziert eine „grammar of action“, dies tun auch Applikationen wie Amazon und Facebook, wenn sie festlegen, welche Aktivitäten zulässig sind und wie sie kombiniert werden können (ausführlicher Gerlitz 2016).
} 
places online, nor does it categorize existing social activities (we do not usually ,follow ' friends offline). Rather, encoding creates the actions which users are invited to perform and records the performance of such actions into distinct data fields" (Alaimo und Kallinikos 2017, S. 177; Hervorhebung BH). Dies bedeutet gleichzeitig, dass Handlung und Beobachtung konvergieren. Während bei einer konventionellen Überwachung eine Handlung geschieht und diese erst im Nachhinein beobachtet wird, fallen Handlung und Beobachtung nun zusammen: Jede Aktivität wird unmittelbar als Datenpunkt registriert (Gerlitz 2016, S. 25; Brayne 2021 in diesem Band). Das Operieren von Recommendersystemen gleicht insofern einem geschlossenen Kreislauf, einer Feedbackschleife, die ständig in Bewegung ist. Als Input braucht es zwar die Aktivitäten der Nutzer, aber diese Aktivitäten bewegen sich in den vom System vordefinierten Bahnen. Sobald Nutzer eine Website betreten, hinterlassen sie die Daten, aus denen die Empfehlungen berechnet werden. Diese Empfehlungen können ihrerseits Reaktionen auslösen, z. B. einen neuen Kauf oder ein neues „liken“, die wiederum als Daten in das System eingehen und neue Berechnungen und Empfehlungen initiieren. Damit schafft sich das System auch seine eigenen Lernbedingungen: Jedes Anklicken ist ein „Realexperiment“ (Krohn und Weyer 1989), an dem der Algorithmus getestet werden kann.

Um ein Buch, einen Film oder ein Musikstück zu empfehlen, müssen Recommendersysteme die Vorlieben ihrer Nutzer einschätzen können. Dies geschieht dadurch, dass sie die Aktivitäten der Nutzer als implizite Bewertungen interpretieren: Die Tatsache, dass ich Erziehungsbücher für Hunde, aber nicht für Kinder anklicke, weist für Amazon darauf hin, dass ich an Hunden interessiert bin und deshalb wahrscheinlich eher Hundeleinen als Legosteine kaufen werde. Welche Bedeutung die Nutzer selbst mit ihren Aktivitäten verbinden - ob das Drücken eines Share-Buttons tatsächlich Zustimmung markiert oder ein ,like“ für alle dasselbe meint -, spielt für das System keine Rolle. In der algorithmischen Welt der Recommendersysteme gibt es nur formal definierte Objekte und Aktivitäten, die diese Objekte miteinander verbinden: „Things or entities such as users, comments, photos, posts are all classified as data objects and every activity connecting two objects as action" (Alaimo und Kallinikos 2017, S. 178; Hervorhebung durch die Autoren). Das heißt Nutzer werden über ihre Relation zu Dingen (z.B. kaufen) oder zu anderen Nutzern (z.B. ,folgen“) definiert, und diese über ihre Relation zu den Nutzern (ausführlicher Unternährer 2021a in diesem Band). Individuen haben keine eigene ontologische Dignität - Menschen und Dinge sind austauschbar. In diesem Abstrahieren von den subjektiven Sinngehalten gründet auch die Effizienz von Recommendersystemen. Denn indem sie jedes Liken oder jedes Markieren als identisch behandeln, stellen sie auf einer basalen Ebene Vergleichbarkeit her. „Defining an individual user as an aggregation of likes immediately renders the individual qua likes commensurable to other individuals qua likes“ (Alaimo und Kallinikos 2017, S. 179).

\section{2 „Ich bin die Anderen“- die Operationsweise von Recommendersystemen}

Eine weitere Besonderheit von Recommendersystemen, die auf dem Verfahren des Collaborative Filtering beruhen, liegt darin, dass die Empfehlungen zwar personalisiert sind, die Personalisierung aber nicht aus der persönlichen Nutzergeschichte, 
sondern aus den Aktivitäten anderer Nutzer abgeleitet ist. Das Subjekt, an das sich die Empfehlung richtet, ist im System absent. Abgesehen davon, dass es nur in Form von Datenspuren existiert, sind es die Verhaltensdaten anderer Nutzer und Nutzerinnen und nicht das individuelle Verhaltensprofil, die die Grundlage für die algorithmische Konstruktion von „Personen“ bilden. Ich mag mich zwar als singuläres Individuum adressiert fühlen, das System operiert aber nicht über Singularisierung, sondern über den Vergleich mit der Masse der Anderen.

Collaborative Filtering ist ein algorithmisches Verfahren, das davon ausgeht, dass Nutzer, die einige Vorlieben teilen, auch andere Vorlieben teilen: ,people like you also liked ...": ,,being like“ und ,liking“ werden damit gleichgesetzt (Seaver 2012, k. S.). Die Aufgabe besteht entsprechend darin, durch einen Vergleich meiner Präferenzen mit jenen aller anderen Nutzer jene Nutzer herauszufiltern, die ähnliche Interessen und Vorlieben haben - ein ähnliches Produkt gekauft, die gleiche $\mathrm{Mu}$ sik gehört oder die gleichen Filme ähnlich bewertet haben. Anstatt Empfehlungen aus soziodemografischen Merkmalen abzuleiten, wie es die klassische Marktforschung und stereotypisierende Recommendersysteme tun (Unternährer 2021a in diesem Band), werden sie beim Collaborative Filtering über das Verhalten der Nutzer erschlossen. Die Berechnung von Empfehlungen geschieht grob gesagt in drei Schritten, die Vergleich, Kategorienbildung und Bewertung involvieren. Diese drei Schritte lassen sich zwar analytisch unterscheiden, empirisch sind sie aber ineinander verzahnt und werden bei jedem neuen Click, jedem neuen Kauf und jeder neuen Bewertung wieder in Gang gesetzt (für das Folgende instruktiv Unternährer 2020; Alaimo und Kallinikos 2021).

Im Einzelnen geschieht das Filtern zunächst darüber, dass meine Aktivitäten paarweise mit den Aktivitäten aller anderen Nutzer verglichen werden. Das heißt für jeden Nutzer (oder für jedes Objekt) wird auf der Basis seiner Aktivitäten ein Korrelationskoeffizient berechnet, der angibt, wie ähnlich er allen anderen Nutzern ist. Formal lassen sich diese Vergleiche als eine Matrix darstellen, in der die Nutzer in der Vertikalen und die Produkte in der Horizontalen aufgeführt sind. In den Zellen stehen Ziffern, die die aus den Nutzeraktivitäten erschlossene Bewertung eines Produkts wiedergeben, z. B. eine 1, 2 oder 3 oder auch eine 0 , wenn für den betreffenden Nutzer noch keine Informationen vorliegen. Diese Nutzervergleiche bilden die Grundlage dafür, jene Nutzer, deren Bewertungen meinen am ähnlichsten sind, herauszufiltern. Um zwischen ,people like me“ und ,people unlike me“ zu unterscheiden, werden Grenzwerte festgelegt, z. B. ein bestimmter Korrelationskoeffizient, der gegeben sein muss, um Nutzer als „Nachbarn“ (ein Feldbegriff) zu kategorisieren. ${ }^{5}$ Diese Einstufung ist allerdings flüchtig - bei jeder neuen Aktivität können sich die Nachbarn wieder ändern. Die Kategorisierung ist der Ausgangs-

\footnotetext{
5 Eine Alternative zu diesem nutzerbasierten Vorgehen sind itembasierte Verfahren, wie sie z. B. Amazon verwendet (Smith und Linden 2017). Bei itembasierten Verfahren werden nicht Nutzer, sondern Objekte (Produkte, Artikel, Musikstücke) nach ihrer Ähnlichkeit verglichen und kategorisiert. Ähnlichkeit wird in diesem Fall darüber berechnet, ob sie oft gemeinsam gekauft oder gehört werden. So könnten z. B. Hundeleinen, Regenmäntel und Stoffspielzeuge als ähnlich kategorisiert werden, weil sie von vielen Nutzern immer wieder gemeinsam gekauft wurden. Aber auch in diesem Fall bildet das Verhalten der Nutzer - ihre Kauf- oder Hörgewohnheiten - die Grundlage für die Kategorisierung (ausführlicher Alaimo und Kallinikos 2021).
} 
punkt für den dritten Schritt - die Berechnung der Empfehlungen auf der Grundlage dessen, wie meine Nachbarn Dinge bewertet haben, die ich selbst noch nicht gesehen oder bewertet habe. Die Nutzer, die mir ähnlicher sind als andere, erhalten ein größeres Gewicht und damit einen größeren Einfluss bei der Bestimmung der Produkte, die mir empfohlen werden. Die nominale Kategorie der „Nachbarn“ wird mit anderen Worten intern ordinalisiert. Bewertungen kommen bei diesen Berechnungen in unterschiedlicher Form zum Zuge: zum einen in Form der Nutzerbewertungen, die die Grundlage für die Berechnung von Empfehlungen bilden. Zum anderen ,bewertet" das System aber auch selbst, indem es die Nutzer nicht nur miteinander vergleicht, sondern sie zusätzlich nach ihrer Ähnlichkeit zu mir in eine ordinale Reihung bringt. ${ }^{6}$

Die auf diese Weise errechneten Nutzerkategorien sind rein formal auf der Basis von Ähnlichkeitsmaßen definiert, sie haben zunächst keine inhaltliche Bedeutung. Um sie zu Werbezwecken zu nutzen, müssen sie interpretiert und in die Kategorien des Marketings übersetzt werden. Während klassische Segmentierungstechniken Kundenpräferenzen aus soziodemografischen oder aus Lebensstilmerkmalen erschließen, eröffnet das Verfahren des Collaborative Filtering ganz neue Möglichkeiten zielgruppenspezifischer Werbung. Anstatt Kundinnen nach soziodemografischen Merkmalen zu kategorisieren und die Werbung darauf auszurichten, sortieren sie Nutzer nach der Ähnlichkeit ihres Verhaltens - ihrer Hörgewohnheiten oder ihres Kauf- und Suchverhaltens - und leiten daraus die kategoriale Zugehörigkeit ab (Alaimo und Kallinikos 2019; Cheney-Lippold 2011; Prey 2018). Im Vergleich zur Verfahrensweise der amtlichen Statistik (vgl. Abschn. 3.2) wird das Verhältnis von kategorialer Zugehörigkeit und Verhalten gewissermaßen auf den Kopf gestellt: „,Hispanic-ness“ is performed into being through music listening behavior. If a listener's listening behavior begins to change, so will her Hispanic-ness. The listener may eventually be assigned another ethnic identity“ (Prey 2018, S. 1094). Auf diese Weise werden nominale kategoriale Zugehörigkeiten gradualisiert und kategoriale Zuordnungen werden fluide. Wer als „Hispanic“ kategorisiert wird, ist nicht notwendig jemand, der offiziell als Hispanic eingestuft wurde oder sich auch selbst so kategorisieren würde, sondern eine Person, die Interessen hat, die das System auf der Basis seiner Hörgewohnheiten als „hispanisch“ klassifiziert. Kategoriale Zugehörigkeit schrumpft damit zu einer statistischen Größe, die sich ständig ändern kann (Fisher und Mehozay 2019). „Weiblich“ oder „,hispanisch“ ist das, wofür sich die als „Frau“ oder als „Hispanics“ kategorisierten Nutzer zu einem bestimmten Zeitpunkt interessieren (zum technischen Verfahren Cheney-Lippold 2017, S. $108 \mathrm{ff}$.; Quantcast 2014). Kategorien wie Geschlecht oder Hautfarbe werden dadurch zwar de-essentialisiert, aber sie bleiben weiterhin mit stereotypisierten Verhaltenserwartungen assoziiert. Nur beruhen diese auf statistischen Zusammenhängen und nicht mehr auf kulturellen Schemata.

Cheney-Lippold (2011) spricht in diesem Zusammenhang von einem „cybernetic categorization system“ und macht mit diesem Begriff deutlich, dass die Sortierung

\footnotetext{
6 Algorithmen können natürlich nicht in einem strengen Sinn bewerten: das Bessere vom Schlechteren, das Schönere vom Hässlicheren unterscheiden. Sie bewerten aber insofern, als sie Ereignisse in eine Rangfolge bringen, sie ordinalisieren (dazu auch Fourcade und Healy 2017).
} 
in unterschiedliche Geschlechter, Ethnien oder Altersgruppen nicht über soziale Zuschreibungsprozesse, sondern über algorithmische Berechnungen verläuft. Dass sich im Internet ein eigenes Kategorisierungsuniversum etabliert, stellt für die herkömmliche Forschung zur Personenklassifikation eine Herausforderung dar. Zum einen relativiert es die Annahme, dass die kategoriale Sortierung von Personen eine grundsätzlich soziale Leistung ist. Zum andern wirft es die Frage auf, wie sich die beiden Modi der Personenkategorisierung zueinander verhalten. Inwiefern verändert die ,algorithmic identity“ (Cheney-Lippold 2011) auch das realweltliche Verständnis von „,weiblich“, „,männlich“ oder ,hispanisch“? Und inwieweit hat das zunehmende „crossing“ ehemals fixer kategorialer Grenzen (Brubaker 2016) auch damit zu tun, dass sich die algorithmisch zugeschriebene Identität ständig ändert (vgl. Abschn. 4)? Die Nutzer erfahren zwar nicht explizit, wie sie kategorisiert werden, aber aus den Empfehlungen, die sie erhalten, können sie unter Umständen herauslesen, wie oder als wer sie beobachtet werden. Die persönliche Adressierung legt es nahe, Empfehlungen als Spiegelungen dessen zu begreifen, was ich bewusst oder unbewusst im Netz über mich offenbart habe. Ähnlich wie Empfehlungen können auch solche Spiegelungen als Anreiz fungieren, sich am digitalen „Maussian bargain“ (Fourcade und Kluttz 2020) zu beteiligen und dem Unternehmen auch in Zukunft Daten zur Verfügung zu stellen. ${ }^{7}$

\subsection{Personalisierung - ,,Du“ bin nicht ,Ich“““}

Obschon Recommendersysteme mich in einer personalisierenden Sprache als singuläres Individuum adressieren - „das könnte Dich auch interessieren“, ,,das könntest Du auch mögen“ - ist dieses „Du“ gerade nicht über seine Besonderheit definiert. Das „Du“ ist eine Ansammlung von Datenspuren, und seine Kontur gewinnt es durch den Vergleich mit anderen Nutzern. Personalisierung erfordert mit anderen Worten Entpersonalisierung. Das System weiß nichts über die Individuen, deren Daten es aufzeichnet und die es als konkrete Personen - als „unique cultural individuals“ (Alaimo und Kallinikos 2019, S. 408) - anspricht. Es verfügt nur über Algorithmen, die in den Daten nach Mustern suchen. Das „,Du“ ist mit anderen Worten kein Individuum im klassischen Sinne, sondern ein algorithmisches Konstrukt - ,,an endlessly sub-dividable ,dividual“" (Cheney-Lippold 2011). Lury und Day (2019, S. 20) sprechen in diesem Zusammenhang von einer ,a-typical individuation“: „This pathway (of a-typical individuation) creates ,a' person or individual that is always provisional and corresponds only partially with the type or category in which it is included, whether this concerns what a person might buy, like, share or possess. The term ,pathway " is intended to capture this category of person, a category that is never static but always changing and always in motion".

Insofern ist die These, dass das Internet zu einem Singularisierungsschub führe, nicht so treffend, wie sie auf den ersten Blick vielleicht erscheint. An der Oberfläche mag sie wohl stimmen: Individuen treffen auf digitale Umwelten, in denen sie als unverwechselbare Persönlichkeiten adressiert werden, und stellen sich dort auch so dar. Auf der algorithmischen Hinterbühne sieht es jedoch anders aus. Dort

\footnotetext{
7 Ich danke Markus Unternährer für diesen Hinweis.
} 
werden Subjekte nicht als einzigartige fabriziert und dort findet man auch keine „maschinelle Singularisierung“ (Reckwitz 2017, S. 253 ff.). Vielmehr ergibt sich das ,Subjekt“, das Recommendersysteme ansprechen, über eine massive Standardisierung der Verhaltensoptionen (Abschn. 2.1) und über den Vergleich mit Anderen (Abschn. 2.2). Es sind die Anderen, die die Grundlage dafür bilden, den Einzelnen persönlich zu adressieren. Das „Du“ errechnet sich mit anderen Worten aus seiner Ähnlichkeit mit dem, was die Anderen tun (dazu auch Mennicken und Kornberger 2021 in diesem Band). Der durch personalisierte Empfehlungen erzeugte Eindruck, dass digitale Technologien Subjekte in ihrer Besonderheit zu begreifen versuchen und dadurch den Trend zur Singularisierung verstärken, sollte mit anderen Worten nicht zum Nennwert genommen werden. Das algorithmisch konstruierte Subjekt ist weder einzigartig noch ist es stabil. Als einzigartig erscheint es nur auf der Bildschirmoberfläche. Singularisiert sind die Individuen mit anderen Worten nur auf der Ebene der Darstellung, auf der Ebene der Herstellung sind sie es gerade nicht. So gesehen lässt sich die These auch umkehren. Recommendersysteme singularisieren nicht, aber sie machen sich den Wunsch nach Einzigartigkeit zunutze, um ihre Berechnungen in eine ,subjekttaugliche“ Kommunikationsform zu bringen.

\section{Die Observierung der Gesellschaft - das Beispiel der amtlichen Statistik}

Digitale Beobachtungsinstrumente sind die vorläufig letzte Entwicklung in der Geschichte moderner Beobachtungsformate, die mit der amtlichen Statistik ihren Anfang nahm. Worin unterscheiden sich die Beobachtungstechniken der amtlichen Statistik von jenen digitaler Beobachtungsformate und was sind die Gemeinsamkeiten? Dieser Frage werde ich mich im Folgenden am Beispiel der Bevölkerungsstatistik als dem prominentesten Zweig der amtlichen Statistik zuwenden. ${ }^{8}$ Um die Bevölkerungsstatistik historisch einzuordnen, werde ich zunächst den Kontext kurz umreißen, in dem die moderne Statistik entstand und ihre Zwecksetzung fand. Das zweite Unterkapitel abstrahiert von diesem historischen Kontext und legt den Fokus auf das doing statistics: Wie gelangt die amtliche Statistik zu Daten, wie werden aus diesen Daten Zahlen und inwieweit sind dabei auch Kategorisierungs-, Vergleichsund Bewertungsakte involviert? Das Erstellen einer Bevölkerungsstatistik beginnt mit der Identifizierung der zu zählenden Individuen und verlangt, dass diese sich selbst in die vorgegebenen statistischen Kategorien einordnen. Auf die damit verbundene Doppelrolle der Statistik als Objektivierungs- und Subjektivierungsinstanz gehe ich abschließend ein.

Die technischen Verfahren der heutigen amtlichen Statistik sind nicht mehr dieselben wie zu ihrer Entstehungszeit. Anstatt die Bevölkerung durch Volkszähler zu befragen, werden zunehmend Online-Fragebögen verwendet, anstatt Vollerhebungen durchzuführen, werden teilweise Stichprobenverfahren eingesetzt, und anstatt die In-

\footnotetext{
8 Wenn ich im Folgenden von ,,amtlicher Statistik“ spreche, meine ich damit ausschließlich ihren deskriptiven Teil, also die Erhebung und Komprimierung von Daten, die am Ende in Form von Tabellen und einfachen Maßzahlen dargestellt werden.
} 
formationen aus den Fragebögen von Hand einzutragen und auszuwerten, werden sie elektronisch erfasst und mithilfe von Statistikprogrammen ausgewertet. Trotz dieses technischen Wandels ist ihre Grundlogik aber im Wesentlichen dieselbe geblieben: Festsetzung von Kategorien, Operationalisierung in Form von Fragen, Kodierung, Aggregierung und deskriptive Analysen. Im Folgenden geht es um die Kontinuität dieser Operationslogik, nicht um den Wandel der technischen Verfahren.

\subsection{Die Geburt der Gesellschaft aus dem Geist der Statistik}

Viele Darstellungen zur Geschichte der modernen Statistik lassen diese mit der Einrichtung statistischer Ämter in der ersten Hälfte des 19. Jahrhunderts beginnen. Wie Lars Behrisch (2016) in seiner großangelegten Studie zur Entstehung der modernen Statistik nachweist, lag der entscheidende Wendepunkt jedoch nicht in einer organisatorischen, sondern in einer epistemologischen Transformation, die er auf das späte 18. Jahrhundert datiert und die zu einer grundlegend neuen Auffassung der Bedeutung und des Potenzials von Zahlen führte. ${ }^{9}$ Während sich die früheren Erhebungen auf punktuelle Zählungen beschränkten und die Zahlen lokal für administrative Zwecke einsetzten, waren die Begründer der Statistik nicht mehr an Einzelinformationen interessiert, sondern an der Aufdeckung der Ordnungsmuster, die sich in der Masse der Daten verbargen. Man zählte, um mit den Zahlen rechnen zu können - um sie zu aggregieren, zu korrelieren und über Zeiten und Räume hinweg zu vergleichen. Statistische Erhebungen sollten der „höhern Speculation“ dienen, wie es ein früher Wegbereiter der modernen Statistik formulierte, und nicht durch den ,rohen Gedanken“ motiviert sein, Steuern abzuschöpfen oder Soldaten auszuheben (Franz Seraph von Kohlbrenner 1768, zit. in Behrisch 2016, S. 211). Das entscheidend Neue an der modernen Statistik war mit anderen Worten nicht die zunehmende Verfügbarkeit und Zirkulation von Zahlen. Die ,,avalanche of printed numbers“ (Hacking 1982) war lediglich eine wichtige Voraussetzung. Entscheidend war, dass die Zahlen auf neue Weise interpretiert wurden, nämlich als Hinweis darauf, dass das Soziale einen eigenständigen Realitätsbereich darstellt und eigenen Gesetzen folgt.

Ein wichtiger Wegbereiter und Popularisierer dieser Idee war Adolphe Quetelet, der die Bevölkerung nicht mehr als Summe von Einzelindividuen begriff, sondern als eine Realität sui generis, die eigenständige Gesetzmäßigkeiten aufweist (ausführlicher Heintz 2012, S. 15 ff.). Was sich auf der individuellen Ebene als kontingent als willkürliche „Laune des Willens“ (Quetelet 1838, S. 5) - präsentiere, erweise sich auf der Aggregationsebene des Sozialen als regelmäßig und geordnet. Um die sozialen Gesetze herauszufinden, müsse man, so Quetelet, ,vom einzelnen Menschen abstrahiren. ... Indem wir ihn seiner Individualität entkleiden, beseitigen wir Alles, was zufällig ist“ (Quetelet 1838, S. 3). Auf diese Weise gelange man zum „Durchschnittsmenschen“ - dem homme moyen -, einem „fingirten Wesen“, dessen numerisches Profil die Durchschnittsmerkmale einer Bevölkerung zum Ausdruck bringt (Quetelet 1838, S. 9, 15). Mit seiner These, dass (nationale) Gesellschaften

\footnotetext{
9 Ein wichtiger Vorläufer war die von William Petty und John Graunt bereits in den 1660er-Jahren begründete „Politische Arithmetik“, die aber lange Zeit ein Solitär blieb (Behrisch 2016, S. 34 ff.; Desrosières 2005, S. 26 ff.).
} 
eigenständige Makroeigenschaften aufweisen, vermachte Quetelet den Sozialwissenschaften ein neues Untersuchungsobjekt, das von Emile Durkheim 60 Jahre später als ultimativer Beweis für die Unverzichtbarkeit der Soziologie ins Feld geführt wurde: „Man sieht nicht, dass es keine Soziologie geben kann, wenn es keine Gesellschaften gibt, und dass es keine Gesellschaften gibt, wenn es nur Individuen gäbe“ (Durkheim 1983/1897, S. 21). In Abwandlung eines Aufsatztitels von Friedrich H. Tenbruck (1990) könnte man deshalb auch von der „Geburt der Gesellschaft aus dem Geist der Statistik“" sprechen.

Michel Foucault (2004) verbindet die Entstehung der modernen Statistik mit einer neuen Technik des Regierens, die nicht mehr auf Einschränkung, sondern auf Ermöglichung, nicht mehr auf Disziplinierung, sondern auf Regulierung setzt. Im Mittelpunkt steht nicht mehr der einzelne Untertan, sondern die Bevölkerung, die im 18. Jahrhundert als neue politische Figur auftaucht und die man in ihrer Zusammensetzung und ihren Merkmalen erforschen und vermessen muss, um sie regulierend regieren zu können (Foucault 2004, S. 102 ff.). Dieser Wille zum „Genau-wissenwollen“ (Foucault 2004, S. 114) ist für ihn das entscheidende Moment der genannten epistemologischen Transformation. Während die Disziplinartechniken auf den „Körper-Menschen“ zentriert waren, den es zu kontrollieren und zu dressieren galt, richtet sich die Biopolitik der Bevölkerung auf den „Gattungs-Menschen“ (Foucault 2001, S. 286). Anstatt den Einzelnen zu verändern, geht es nun darum, ihn, wie es Adolphe Quetelet (1838, S. 2) formulierte, ,nur als Bruchtheil der ganzen Gattung“ zu betrachten und zu erforschen, welche überindividuellen Regelmäßigkeiten sich entdecken lassen und welche Ursachen ihnen zugrunde liegen. Mortalitäts- und Geburtenraten lassen sich nicht durch Dekrete steuern, Suizide nicht durch Individualkontrollen verhindern, Ernteerträge nicht durch Gesetze erhöhen. Doch sobald man in der Lage ist, jenseits der Zufälligkeiten des individuellen Geschehens die sozialen Muster zu erkennen, wird es möglich, auf sie einzuwirken. Dieses Wissen zu beschaffen, war die Aufgabe der modernen amtlichen Statistik.

Bis die epistemologischen Ziele der frühen Statistiker in der amtlichen Statistik, die riesige Datenmengen zu erheben und zu kompilieren hatte, auch praktisch umgesetzt werden konnten, dauerte es allerdings lange. In den meisten europäischen Ländern wurden die Ergebnisse noch lange als Listen veröffentlicht, die die Informationen nur nach Rubriken geordnet präsentierten. Erst in der zweiten Hälfte des 19. Jahrhunderts wurden technische Verfahren entwickelt, die es auch im Falle von Massendaten erlaubten, die Informationen nicht nur entlang einzelner Kategorien auszuzählen, sondern die Kategorien selbst zueinander in Beziehung zu setzen und die Ergebnisse in Form von Tabellen darzustellen (von Oertzen 2017a; Schneider 2013, S. 238f.). Die entscheidende Neuerung bestand in der Einführung einer ,neuen Papiertechnologie“ (von Oertzen 2017a, S. 424), des sogenannten Zählblättchens, mit dessen Hilfe sich Kategorien auf relativ effiziente Weise miteinander verknüpfen ließen. ${ }^{10}$ Dies geschah dadurch, dass zunächst Hauptstapel gebildet wurden, z. B.

\footnotetext{
10 Eine weitere technische Innovation war die sogenannte Zählkarte, die ähnlich funktionierte wie die Zählblättchen (zur Zählkarte auch Wobbe 2021 in diesem Band). Die Neuerung bestand darin, dass die Zählkarte gleichzeitig als Fragebogenformular fungierte, in das die Antworten direkt eingetragen werden konnten. Dadurch wurde der Schritt der Datenübertragung überflüssig.
} 
getrennt nach Geschlecht. Diese beiden Stapel konnten die „Auszähler“ in einem zweiten Schritt nach einem weiteren Kriterium, z.B. der Altersklasse, in kleinere Stapel aufteilen. Durch das Auszählen dieser Stapel erhielt man Werte für jede Subkategorie, die in eine Tabelle eingetragen wurden, die die Häufigkeitsverteilungen wiedergab: Altersgruppen ließen sich nun nach ihren Geschlechteranteilen und Frauen und Männer nach ihrer Altersverteilung vergleichen. Erst mit dieser Innovation wurde es auch für große Datenmengen möglich, einen Einblick in die strukturellen Zusammenhänge zu gewinnen, die die Statistiker seit Ende des 18. Jahrhunderts so interessiert hatten. ${ }^{11}$

\subsection{Doing statistics - die Fabrikation statistischer Zahlen}

Statistiken beanspruchen zwar, eine ihnen äußerliche Welt abzubilden, es fehlt ihnen aber eine von ihren epistemischen Vorleistungen und Verfahren unabhängige Referenz. Im Unterschied zu digitalen Technologien (Abschn. 2.1) generieren sie zwar nicht selbst die Objekte, die sie vermessen, aber am Anfang stehen nicht extern gegebene Sachverhalte, sondern Konventionen und Praktiken, über die die Objekte der Statistik erst hergestellt werden. Auch statistische Daten sind mit anderen Worten fabriziert. „Raw data is an oxymoron“, wie es im Titel eines einschlägigen Sammelbandes heißt (Gitelman 2013).

Aber wie werden die Daten erzeugt, und wie werden aus diesen Daten Zahlen? Um diese Fragen zu beantworten, muss man die konkreten Praktiken der Zahlenproduktion in den Blick nehmen - das doing statistics. Zu diesem Thema liegen allerdings kaum Arbeiten vor. Seit Karin Knorr Cetina (1984) und Bruno Latour und Steve Woolgar (1979) das Genre der Laborstudien begründet haben, sind eine Vielzahl von Untersuchungen erschienen, die Wissenschaftlerinnen und Wissenschaftlern bei ihrer praktischen Forschungsarbeit über die Schultern schauen, aber meines Wissens gibt es keine Studie, die dasselbe bei Statistikern tut. ${ }^{12}$ Die wenigen Untersuchungen, die nicht von bereits fabrizierten Zahlen ausgehen, sondern sich für deren Zustandekommen interessieren, schließen darauf aus schriftlichen Dokumenten wie Handbüchern, Fragebögen, Kodieranweisungen oder Ähnlichem (vgl. etwa Cakici et al. 2020; Beiträge in Supik und Spielhaus 2019; Brückweh 2015, Kap. II). ${ }^{13}$

Statistische Zahlen sind das Endprodukt eines komplexen Herstellungsprozesses, der verschiedene Phasen durchläuft und den ich im Folgenden vor allem am Beispiel der Befragung und der Kodierung veranschaulichen möchte (ausführlicher Bennani

\footnotetext{
11 Das Zählblättchen-Verfahren war eine Art manuelles Äquivalent zur Hollerith-Maschine, die im USZensus zum ersten Mal 1890 eingesetzt wurde. Beide funktionierten nach demselben Prinzip, nur wurden im einen Fall menschliche „Computer“ eingesetzt, im anderen Fall Maschinen (von Oertzen 2017b).

12 Ausnahmen sind Jerven (2013) und Biruk (2018). Ihr Fokus liegt jedoch auf den Feldpraktiken von Statistikern und ihr Untersuchungsbereich sind Länder, in denen es noch keine etablierte statistische Infrastruktur gibt.

13 Daneben gibt es natürlich viele Lehrbücher zur empirischen Sozialforschung und den Methoden der Statistik. Sie haben aber eine präskriptive Ausrichtung und vermitteln nur am Rande einen Einblick in die Alltagspraktiken von Statistikern. Sich auf sie zu stützen, wäre so, wie wenn man die Forschungsarbeit im Labor auf der Basis von wissenschaftstheoretischen Handbüchern rekonstruieren wollte.
} 
und Müller 2021 in diesem Band). Für Alain Desrosières sind die der eigentlichen Messung vorauslaufenden Konventionalisierungen, also die Festlegung von Kategorien und die Entscheidungen über Indikatoren, Variablen und Kodierregeln, das Kernstück der Quantifizierung: „The word ,measure“ is misleading and leaves conventions of quantification unexamined. The verb ,to quantify', in its active form (to make into a number), supposes that a series of hypothetical equivalences has been developed and made explicit ... Measurement itself comes after that, as the rule-based implementation of these conventions. From this point of view, quantification breaks down into two successive parts: convention and measurement" (Desrosières 2015, S. 333). Mit seiner Unterscheidung zwischen Konventionalisierung und Messung und der Annahme, dass Quantifizierung mehr umfasst als bloße Messung, grenzt sich Desrosières deutlich von den meisten anderen Autorinnen und Autoren ab, die Quantifizierung mit Messen gleichsetzen und damit den Fokus auf den Messvorgang legen, der für Desrosières gegenüber den sozialen Prozessen seiner Ermöglichung sekundär ist. ${ }^{14}$

„Counting is hungry of categories“, schreibt Ian Hacking (1982, S. 280). Denn um zu zählen, muss das, was gezählt wird, zuerst vergleichbar gemacht werden. Dies geschieht über die Festlegung von Kategorien oder „Äquivalenzkonventionen“ (Desrosières 2005). Was „draußen“ vorliegt und was Statistiken abzubilden beanspruchen, sind singuläre und situierte Sachverhalte. Um zu einer Zahl zu werden, müssen sie kategorial vereinheitlicht werden: Gemeinsames wird akzentuiert, Unterschiede werden ausgeblendet. Obschon sich Frauen in vielerlei Hinsicht unterscheiden, werden alle unter Ausblendung ihrer sonstigen Unterschiede unter den gemeinsamen Nenner „Frauen“ gebracht und gleichzeitig von den „Männern“ abgegrenzt. Kategorien liefern mit anderen Worten, was Statistiken brauchen - sie vereinheitlichen und separieren in einem Zug (Zerubavel 1996). Dem eigentlichen Mess- und Zählvorgang geht also die Festlegung eines kategorialen Ordnungsgerüsts voraus, das die Vergleichbarkeit dessen, was gezählt werden soll, erst herstellt. Demgegenüber kommen Recommendersysteme, die auf Collaborative Filtering beruhen, ohne kategoriale Vorentscheidungen aus: Kategoriale Zugehörigkeiten werden nicht vorausgesetzt, sondern aus dem Verhalten erschlossen. Vergleichbarkeit wird nicht über Kategorienbildung und Kodierung erzeugt, sondern darüber, dass sie standardisierte Verhaltensoptionen vorgeben, die für die Nutzer Unterschiedliches bedeuten können, vom System aber als identisch behandelt werden (vgl. Abschn. 2.1).

Aber auch wenn die den Messvorgang fundierenden Konventionen festgelegt sind, bleibt immer noch viel Raum für Entscheidungen, die sich nicht von vornherein festlegen lassen. Denn der Messvorgang ist eben keine ,rule-based implementation of conventions“ (Desrosières 2015, S. 333), sondern vollzieht sich als situiertes praktisches Handeln. Wie komplex und sozial imprägniert der Weg von den „Dingen“ zu den „Zahlen“ ist, belegt die Durchführung statistischer Befragun-

\footnotetext{
14 In gewissem Sinne setzen auch digitale Technologien „Konventionalisierung“ voraus. Bevor die Daten algorithmisch prozessiert werden, muss z. B. festgelegt werden, welche Bedingungen die aufgezeichneten Aktivitätsspuren erfüllen müssen, um als Daten encodiert zu werden, und es müssen Entscheidungen darüber getroffen werden, von welchen Schwellenwerten an Nutzer als „Nachbarn“ kategorisiert werden (Alaimo und Kallinikos 2021).
} 
gen. Das Ausfüllen der Fragebögen ist ein grundsätzlich sozialer Prozess, der bis zur Umstellung auf postalische Befragungen oder Online-Fragebögen einen direkten Kontakt zwischen Interviewern und Befragten voraussetzte, sei es bei der Kontaktaufnahme oder beim Ausfüllen der Fragebögen und ihrer Erläuterung (anschaulich Brückweh 2015, S. 117 ff.; Igo 2007, Kap. 3; Ruppert 2009a). ${ }^{15}$ Ein standardisiertes Interview unterscheidet sich zwar von einer alläglichen Konversation, aber es bleibt ein interaktives Geschehen, das für seinen Erfolg darauf angewiesen ist, dass die Beteiligten ihre alltäglichen Interaktionskompetenzen einbringen (Suchman und Jordan 1990; Maynard und Schaeffer 2000). Schriftliche Befragungen vollziehen sich zwar ohne direkten Kontakt, aber mit ihrer Frage-/Antwort-Struktur evozieren auch schriftliche Fragebögen eine Interaktionssituation, die vonseiten der Befragten ebenfalls Deutungskompetenz und die Fähigkeit zum Perspektivenwechsel erfordert. Die Antworten, die in einem Fragebogen stehen, sind mit anderen Worten nicht einfach objektive Beschreibungen eines Sachverhaltes, sondern sie sind auch Reaktionen auf eine Frage, die in einem faktischen (oder imaginierten) interaktiven Kontext eingebettet ist (Cicourel 1970, S. 110 ff.). Demgegenüber läuft die Datenerfassung bei Recommendersystemen sprach- und interaktionsfrei ab. Es werden keine Äußerungen encodiert, sondern vorgegebene Aktivitäten. Damit entledigen sich digitale Beobachtungstechniken zwar des Problems der doppelten Hermeneutik, die jeder Befragung inhärent ist (Keller 2001, S. $234 \mathrm{ff}$.), aber sie handeln sich dafür eine Vieldeutigkeit ihrer Daten ein (Paßmann und Gerlitz 2014).

Fragebögen sind zwar Messinstrumente, aber sie enthalten noch keine Daten im strengen Sinne. Um aus ihnen Daten und anschließend Zahlen zu gewinnen, müssen die Antworten kodiert, d.h. metrisiert werden. Im Prinzip geschieht dies dadurch, dass den Antworten gemäß eines vorgegebenen Kodeplans Ziffern zugeordnet werden. „Measurement“, so die bekannte Definition von Stanley S. Stevens (1946, S. 677), ,is the assignment of numerals to object or events according to rules“. Manchmal, etwa bei der Kodierung der Geschlechtszugehörigkeit, wirft die Zuordnung von Ziffern keine Probleme auf. Aber überall dort, wo Fragen offen formuliert oder mehrere Antwortmöglichkeiten zugelassen sind, müssen die Antworten für die weitere Auswertung kategorisiert, d.h. übergeordneten Kategorien zugeordnet werden. Was in der Theorie als ein mechanischer Vorgang erscheinen mag, ist in der Praxis ein interpretatives Geschehen. ${ }^{16}$ Kodierregeln sind keine quasikausalen Größen, die ihre Anwendung von vornherein festlegen, sondern sie müssen zusammen mit dem Sachverhalt, den es zu kodieren gilt, gedeutet werden. Wie Garfinkel (1967, S. $18 \mathrm{ff}$.) am Beispiel der Kodierung von Patientenakten zeigt, erfordert diese Deutung situative Entscheidungen und ein Vorwissen darüber, was durch die Kodierung erst erfasst werden soll. In eine ähnliche Richtung weisen auch die Studien, die im Umkreis von Alain Desrosières zur Kodierung von Berufen durchgeführt wurden (Boltanski und Thévenot 1983; Thévenot 2016; Penissat et al. 2016; Diaz-Bone 2018, S. 75 ff.). Auch hier zeigte sich, dass die Kodierer auf ihr alltagstheoretisches

\footnotetext{
15 In Großbritannien und Deutschland wurde schon sehr früh auf schriftliche Befragung umgestellt (Brückweh 2015, Kap. II.2.; von Oertzen 2017a, S. 419), in anderen Ländern und insbesondere bei der Umfrageforschung hielt sich die persönliche Befragung länger.

$16 \mathrm{Zu}$ den Kodierungsverfahren der frühen Statistik von Oertzen (2017a, S. 423).
} 
Wissen über Berufe zurückgriffen und sich zu diesen auch selbst in Beziehung setzten - oder in der Terminologie von Garfinkel (1967, S. 22), the position of a socially competent member of the arrangement" einnahmen. Zusammengenommen belegen diese Untersuchungen, dass das doing statistics, trotz aller Standardisierungsbemühungen, auf die sozialen Fertigkeiten derer angewiesen ist, die das Geschäft der Statistik betreiben, wie auch auf die alltagspraktischen Kompetenzen jener, die von ihnen vermessen werden (vgl. Abschn. 3.3).

Die Kodierung ist ein erster Schritt in Richtung Dekontextualisierung und Abstraktion. Denn durch die Zuordnung der Antworten zu einer vorgegebenen Kategorie werden die Angaben vereinheitlicht und die Befragten untereinander vergleichbar gemacht. Anstatt durch ihren je individuellen Beruf gekennzeichnet zu sein, sind sie nun durch Ziffern repräsentiert, die eine bestimmte Berufskategorie anzeigen. Auf diese Weise erhält jeder und jede ein eigenes numerisches Datenprofil, das sich zusammen mit allen anderen Profilen in eine Datenmatrix eintragen lässt. ${ }^{17}$ Während die Befragten in der Datenmatrix noch je einzeln erkennbar sind, wenn auch nur in Form einer Ziffernkombination, werden die Messwerte in einem zweiten Schritt verdichtet und zu einer Zahl aggregiert, die sich nun auf die von den Einzelindividuen abgelöste Gesamtheit aller Fälle bezieht. Man sieht nun auf einen Blick, wie viele Frauen und Männer, Behinderte und Nichtbehinderte es gibt und kann daraus ihre relativen Anteile ableiten. Was einfach klingen mag, ist faktisch ein anspruchsvoller Vorgang, der aufseiten der Statistiker eine teilweise aufwendige Deutungsarbeit voraussetzt (Mair und Greiffenhagen 2016, S. 60 ff.). ${ }^{18}$ Dies ist besonders dann der Fall, wenn das interessierende Merkmal über eine Skala erhoben wurde (Beispiel Behinderung) oder Mehrfachantworten möglich waren (Beispiel Ethnizität). Um am Ende eine aussagekräftige Zahl zu erhalten, müssen die Häufigkeitsverteilungen verglichen und unter Umständen neue, kompaktere Kategorien gebildet und Schwellenwerte gesetzt werden (Bennani und Müller 2021 in diesem Band). Mit diesem letzten Schritt verschwindet die Vielfalt der individuellen Fälle in einer vereinheitlichenden Zahl, die eine übergeordnete Größe - die Männer, die Behinderten repräsentiert und keine Spuren ihrer Herstellung mehr erkennen lässt. Sobald solche Größen vorliegen, lassen sie sich mit anderen in Beziehung setzen und anhand ihrer Merkmale vergleichen. Die kanonische Präsentationsform dieser Relationierung ist die Tabelle, mit der die Statistik über ein eigenes Kommunikationsformat verfügt. ${ }^{19}$

Im Unterschied zu Listen, die eine lineare und sequenzielle Struktur haben, fügen Tabellen ihre Informationen in ein durch horizontale Zeilen und vertikale Spalten gewonnenes Feldersystem ein und machen auf diese Weise Zusammenhänge im buchstäblichen Sinne sichtbar (anschaulich Segelken 2010, S. $89 \mathrm{ff}$.). Durch die rasterförmige Darstellung werden mindestens zwei Dimensionen grafisch so zueinander

\footnotetext{
17 Die Datenmatrix folgt im Prinzip der gleichen Darstellungsweise wie die von Herrmann Hollerith für den US-Zensus entwickelte Lochkarte. Die Ziffern in einer Zeile entsprechen einer Lochkarte.

$18 \mathrm{Zu}$ den manuellen Sortierungs- und Auszählungstechniken in der Frühzeit der amtlichen Statistik instruktiv von Oertzen (2017a, S. $416 \mathrm{ff}$.).

19 Für das Format ist es an sich unerheblich, ob in den Zellen eine Zahl steht oder ein Wort. Quantitative Tabellen, so wie sie für uns heute selbstverständlich sind, wurden erst im Verlaufe des 19. Jahrhunderts zum Normalfall, vergleiche Desrosières (2005, S. 40 ff.), von Oertzen (2017a) sowie W. Steinmetz 2021 in diesem Band.
} 
in Beziehung gesetzt, dass Unterschiede auf einen Blick erkennbar sind. Diese Darstellungsform bricht die sequenzielle Abfolge von Listen auf und lenkt den Blick in zwei Richtungen, je nachdem ob man die Zeilen liest oder die Spalten. Tabellen, die Geschlecht und Beruf zueinander in Beziehung setzen, ermöglichen, vertikal gelesen, einen Vergleich der verschiedenen Berufe hinsichtlich ihres Männer- oder Frauenanteils und horizontal gelesen einen Vergleich der Geschlechterzusammensetzung der einzelnen Berufe. Im einen Fall ist das Geschlecht das Vergleichskriterium und die Berufsgruppe die Vergleichseinheit, im anderen Fall verhält es sich umgekehrt. Tabellen bringen mit anderen Worten Zahlen in eine Darstellungsform, die den Betrachter automatisch zu einem Vergleich auffordert. „Le tableau croisé ... permet d'exprimer des rapports de rapports, des comparaisons de comparaisons“" (Desrosières 2010, S. 123).

Die in Tabellen aufgeführten Zahlen präsentieren sich als objektiv und neutral. Sie halten Unterschiede und Ähnlichkeiten fest, ohne diese explizit zu bewerten, wie es z. B. Rankings tun. Faktisch gehen in sie aber eine Vielzahl von Bewertungsakten ein (am Beispiel der deutschen Integrationsmonitore Petzke 2021 in diesem Band). Dies geschieht schon dadurch, dass die Statistik über das Konzept der ,Normalverteilung“ den Durchschnitt mit dem „Normalen“ gleichsetzt und das davon Abweichende daran misst. Schon für Alphonse Quetelet repräsentierte der ,homme moyen“ den „Normalbürger“ (s. oben) und Emile Durkheim setzte den „Durchschnittstypus“ mit dem ,normalen Typus“ gleich (Durkheim 1984/1895, S. 148). Für Jürgen Link (2009) besteht deshalb eine enge Verbindung zwischen der Statistik und dem Dispositiv des „Normalismus“. Indem das Durchschnittliche für das Selbstverständliche steht, wird das ihm nicht Entsprechende als das Abweichende und Ungewöhnliche markiert und zum „Normalen“ in eine asymmetrische Relation gebracht (Zerubavel 2018, S. $17 \mathrm{ff}$.).

Auch die Entscheidung für bestimmte Kategorien und Indikatoren (und die Auslassung anderer) hat wertenden Charakter, z. B. wenn im US-Zensus Personen aus der arabischen Welt als „Weiße“ kategorisiert werden (Cainkar 2006) oder wenn Berufstätigkeit über Bezahlung und Regelmäßigkeit operationalisiert wird und somit Frauen, die unbezahlte Familienarbeit leisten, aus der ökonomischen Definition von Arbeit ausschließt (Wobbe 2021 in diesem Band). ${ }^{20}$ Solche vorauslaufenden Bewertungsakte sind in der Präsentation der Daten nicht mehr sichtbar. Was man sieht, sind Zahlen, die keine Spur einer Bewertung mehr erkennen lassen. An dieser Transformation des ,view from somewhere“ zu einem ,view from nowhere“ (Porter 1992, S. 646) hat das Tabellenformat einen erheblichen Anteil. Tabellen sind das Endprodukt einer Purifizierung, mit der sämtliche Vorleistungen - Desrosières' „Konventionalisierungen“ - sukzessiv ausgelöscht werden. Sie haben keinen Autor, dem die Zahlen zugerechnet werden können, und suggerieren damit eine „Position der Exteriorität“ (Desrosières 2005, S. 26). Zahlen werden in Luhmanns Terminologie ,erlebt“, d.h. der Umwelt zugerechnet, und nicht den Entscheidungen jener, die sie erstellt haben (Heintz 2010).

\footnotetext{
20 Als besonders anschauliches Beispiel für den wertenden Charakter statistischer Kategorien vergleiche die Kategorisierungsexperimente von Boltanski und Thévenot (1983).
} 


\subsection{Gesellschaftskörper und Individuum}

Die Bevölkerungsstatistik interessiert sich nicht für das Einzelindividuum und dessen Verhalten, sondern für übergreifende Zusammenhänge. Aber obschon das Individuum am Ende in den Massendaten verschwindet, muss es zuerst namentlich identifiziert und als Teil der Bevölkerung klassifiziert werden. Deshalb konnte die Statistik erst dann auf Dauer gestellt werden, als die grundsätzliche Gleichheit und damit auch Vergleichbarkeit der Einwohner als relevanter eingestuft wurde als ihre sozialen Unterschiede. „It makes no sense to count people if their common personhood is not seen as somehow more significant than their differences" (Porter 1986, S. 25). Über diese basale Gleichheitsannahme zieht die Bevölkerungsstatistik eine kategoriale Grenze zwischen denen, die als Gesellschaftsmitglieder zählen (und folglich gezählt werden), und jenen, die nicht dazu gehören (Renard 2021 in diesem Band). An diesen ersten Klassifikationsakt schließen sich weitere Kategorien an, die das Kerngerüst jeder Bevölkerungsstatistik bilden: Berufskategorien, Einkommenskategorien, ethnische Kategorien, Geschlechterkategorien und viele andere mehr. Alle diese Kategorien unterstellen Vergleichbarkeit und abstrahieren von den individuellen Erscheinungsformen.

Während Individuen für die Statistik nur ein Sprungbrett sind, um die Merkmale des Gesellschaftskörpers aufzudecken, wollen digitale Beobachtungsinstrumente etwas über die Verhaltensmuster konkreter Personen in Erfahrung bringen: Könnte A rückfällig werden? Woran könnte B interessiert sein? Ist C ein potenzieller Täter? Doch auch für sie ist das Individuum nicht eine Person, die es in ihrer Individualität $\mathrm{zu}$ erfassen gilt, sondern ein rechnerisches Konstrukt, das sich aus dem Vergleich mit anderen ergibt (vgl. Abschn. 2.2). Es ist nicht meine höchstpersönliche Datengeschichte, die mich für Recommendersysteme zu einer besonderen Person macht, sondern es ist der Grad an Übereinstimmung (oder Differenz) mit der amorphen Masse anderer Nutzer. Identifizierung ist mit anderen Worten das Ergebnis von Berechnungen und nicht ihr Ausgangspunkt.

Statistiken machen Individuen zu Objekten, die sich zählen und vergleichen lassen. Diese Objektivierung setzt jedoch einen reflexionsfähigen Akteur voraus, der willens und in der Lage ist, sich selbst in die vorgegebenen Kategorien einzuordnen (Ruppert 2009a). Um einen Fragebogen auszufüllen, müssen sich die Befragten in einem Kategorienraster verorten und darüber nachdenken, wer sie sind im Vergleich zu anderen: eine Frau (und kein Mann), verheiratet (und nicht ledig oder geschieden), erwerbstätig (und nicht Hausfrau), Hispanic (und nicht Black). Individuen sind mit anderen Worten nicht bloß passive Objekte der Statistik, die am Ende in den „großen Zahlen“ (Desrosières 2005) verschwinden, sondern sie kategorisieren sich selbst im Spiegel der statistischen Kategorien und machen durch diesen Akt der Selbstidentifizierung ihre statistische Erfassung erst möglich. Evelyn Ruppert spricht in diesem Zusammenhang von einer ,double identification“: „Through the census the subject identifies herself as part of the population and the state identifies the subject and assembles the population“ (Ruppert 2009b, k.S.). Ähnlich wie es Sarah Igo (2007) für die Meinungsforschung gezeigt hat, erzeugen auch Statistiken nicht nur gesellschaftliche Selbstbeschreibungen, sondern sie beeinflussen auch die 
Art und Weise, wie sich Individuen im Vergleich zu anderen wahrnehmen und tragen damit zu deren Selbstkategorisierung und „Subjektivierung“ bei (Hacking 1986).

Wie radikal die amtliche Statistik auf die Selbstidentifizierung der Befragten angewiesen ist, wird in Situationen augenfällig, in denen die Selbstkategorisierung nicht an die kategoriale Ordnung der Statistik anschließbar ist. Dies ist systematisch dann der Fall, wenn das statistische Kategoriensystem auf Kulturen übertragen wird, die in einer anderen kategorialen Ordnung leben, z. B. Kulturen, in denen es keine Berufe, keine Ehen, keine abgrenzbaren Kleinfamilien, keine Marktwirtschaft gibt. Da sich die Befragten in den vorgegebenen Kategorien nicht wiederfinden, können sie die Fragen nicht beantworten und folglich auch nicht klassifiziert werden. Sie bleiben eine Residualkategorie, die sogenannten ,others“, die sich der statistischen Beobachtung entziehen und sich selbst auch nicht als zugehörig wahrnehmen können (exemplarisch Ruppert 2009a). Demgegenüber setzen digitale Technologien für ihre Datengewinnung nicht voraus, dass sich die Nutzer kategorial verorten. Die Individuen bleiben ihnen gegenüber passiv, aber sie hinterlassen durch ihre Aktivitäten Datenspuren, die hinter ihrem Rücken erfasst und verarbeitet werden. Ruppert (2011) spricht deshalb von ,interpassive subjects“ und macht mit dieser Bezeichnung kenntlich, dass Individuen zwar in Netzaktivitäten verwickelt, aber an ihrer Datafizierung nicht aktiv beteiligt sind.

\section{Schlussfolgerungen: Die moderne Gesellschaft im Spiegel ihrer Beobachtungsformate}

Ausgangspunkt meines Beitrags war die Annahme, dass sich digitale Technologien in der Geschichte moderner Beobachtungsformate verorten lassen. Streamingdienste, Suchmaschinen, Vermittlungsplattformen und soziale Netzwerke sind nicht das schlechthin Neue, sondern sie stehen in einer historischen Kontinuitätslinie zu früheren Beobachtungsformaten - der Statistik, der Meinungs- und Marktforschung und dem Sozialmonitoring. Die Gemeinsamkeit liegt darin, dass es sich bei allen um Instanzen handelt, die kontinuierlich Daten erheben und diese Daten nach Regelmäßigkeiten absuchen, um auf diese Weise Informationen zu gewinnen, die sich für weitere Zwecke nutzen lassen. Wie sie das tun und mit welchem Ziel, unterscheidet sich allerdings. Das habe ich anhand eines Vergleichs von Bevölkerungsstatistik und personalisierten Recommendersystemen dargestellt. Verortet man die beiden Beobachtungsformate nach ihrer Entstehungszeit auf einer historischen Zeitachse, gibt der Vergleich nicht nur Aufschluss darüber, wie sie beobachten und worin sich ihre Beobachtungstechnik unterscheidet, sondern auch darüber, wie sich die gesellschaftlichen Grundlagen der Sozialbeobachtung in den letzten 200 Jahren verändert haben.

Mein Vergleich bewegt sich auf einer tieferen Abstraktionsebene als die Theorien des Digitalen, die Armin Nassehi (2019) und Dirk Baecker (2018) vorgelegt haben, und er kommt auch zu anderen Schlüssen. Das Bezugsproblem, von dem Nassehi ausgeht, ist der Komplexitätszuwachs, der mit der Umstellung auf funktionale Differenzierung einhergeht. Die damit verbundene Unübersichtlichkeit schafft einen neuen Bedarf an Planung, Steuerung und Entscheidungshilfen und damit an Tech- 
niken, die in der Lage sind, latente Muster zu entdecken. Die amtliche Statistik ist für ihn eine erste Reaktion auf diese Problemlage, digitale Technologien führen sie weiter. Während Nassehi digitale Technologien nur als Steigerung dessen begreift, was mit der modernen Statistik begann, diagnostiziert Baecker einen grundlegenden Bruch. Anstatt digitale Technologien mit funktionaler Differenzierung kurzzuschließen, sieht er ihr entscheidendes Merkmal darin, dass sie die Grenzen der gesellschaftlichen Funktionssysteme unterlaufen und eine neue Gesellschaftsform vorantreiben, deren Primärstruktur die des Netzwerks ist (Baecker 2018, S. 35 ff.). Im Unterschied zu Nassehi, der die strukturelle Stabilität der modernen Gesellschaft in den Mittelpunkt stellt und sich gegen Thesen einer zunehmenden Verflüssigung gesellschaftlicher Strukturen wendet, verortet Baecker den Bruch darin, dass digitale Technologien ständig neue und grundsätzlich flüchtige Verbindungen herstellen. Das permanente Herstellen und Auflösen von Strukturen ist für ihn ein wesentliches Moment der „nächsten Gesellschaft“ (Baecker 2007).

Die beiden Entwürfe unterscheiden sich in grundsätzlicher Hinsicht. Eine Parallele liegt aber darin, dass sie sich am Erscheinungsbild des Digitalen orientieren und die kleinteiligen technisch-algorithmischen Prozesse, die ihm zugrunde liegen, weitgehend ignorieren. Die Argumente treten zwar mit hohem theoretischen Anspruch auf, sie kommen aber teilweise etwas gar großmundig daher, etwa wenn Nassehi (2019, S. 31) die Statistik (und mit ihr die moderne Gesellschaft) kurzerhand unter „digital“ verbucht und ,digital“ mit ,zählbar“ gleichsetzt oder wenn Baecker (2018, S. 59) die „Digitalisierung der Gesellschaft“ als „,die Erarbeitung und Erprobung abzählbarer und berechenbarer Daten im Medium analoger Widersprüchlichkeit“" definiert. Diese Unschärfen haben Folgen für die Plausibilität ihrer Argumentation. Denn ohne sich in die Niederungen der konkreten Operationsweise digitaler Technologien zu begeben, bleibt sie, wie Passoth und Rammert (2020, S. 314) schreiben, eine „These ohne Test“ - ein theoretisches Programm, das sich um die Phänomene, die es erklären will, zu wenig kümmert.

Mein Beitrag versteht sich als ein erster Schritt zu einem solchen Test, indem ich das, was Nassehi und Baecker unter dem Allgemeinbegriff der Digitalisierung abhandeln, auf einen konkreten Fall herunterbuchstabiere und am Beispiel personalisierter Recommendersysteme aufzeige, wie digitale Technologien konkret operieren. Ich folge der Sichtweise von Nassehi insofern, als auch ich die Kontinuitäten hervorhebe, die zwischen der amtlichen Statistik und digitalen Technologien bestehen, ohne jedoch seinen Funktionalismus zu teilen. Diese Kontinuität habe ich mit dem Begriff des Beobachtungsformats zum Ausdruck gebracht. Gleichzeitig hat mein Vergleich aber auch vielfältige Unterschiede sichtbar gemacht, die es unangemessen erscheinen lassen, die beiden Beobachtungsformate unbesehen in den gleichen Topf zu werfen. Ein wesentlicher Unterschied liegt darin, dass die amtliche Statistik eine Gesellschaft unterstellt, in der sich Zusammenhänge über die Zeit hinweg nicht wesentlich verändern, während Recommendersysteme von verflüssigten Strukturen ausgehen, die sich nur momenthaft stabilisieren, ein Befund, der eher der Gesellschaftsdiagnose von Dirk Baecker entspricht. Auf die Diskussion darüber, ob wir noch in einer funktional differenzierten Gesellschaft leben oder schon in der netzförmig organisierten ,nächsten Gesellschaft“ angekommen sind, will ich mich nicht einlassen. Wer wollte das und vor allem auch wie entscheiden. Stattdessen möchte 
ich ein gesellschaftliches Ordnungsprinzip in den Mittelpunkt stellen, das in der Systemtheorie oft vernachlässigt wird, nämlich die Ordnung, die durch die kategoriale Sortierung von Individuen hergestellt wird, und die Unordnung, die sich ergibt, wenn soziale Kategorien fluide werden. ${ }^{21}$

Die Muster, die die Bevölkerungsstatistik in ihren Daten entdeckt, ergeben sich aus der Festlegung von Kategorien. Über Kategorien werden Individuen vergleichbar gemacht und über Kategorien reduzieren Statistiken die Komplexität, die sie in ihren Daten vorfinden. Dahinter steht die Annahme, dass kategoriale Merkmale Effekte erzeugen, die sich als Regelmäßigkeiten in den statistischen Daten wiederfinden lassen. Was auch immer das individuelle Motiv sein mag, seinem Leben ein Ende zu bereiten - Suizide treten bei Protestanten häufiger auf als bei Katholiken (zumindest zu jener Zeit, als Durkheim seine Studie verfasste). Die konfessionelle Zugehörigkeit scheint offensichtlich einen Einfluss auf die Wahrscheinlichkeit zu haben, Suizid zu begehen. Dieselbe Erklärungslogik lässt sich auch auf andere Verhaltensweisen übertragen. Wer lieber Jazz hört als deutsche Schlager, kann dies aus unterschiedlichen Gründen tun. Aus der Bourdieuschen Kartografie lässt sich jedoch ablesen, dass der Geschmack systematisch mit dem Ort im sozialen Raum variiert. Die Annahme, dass kategoriale Zugehörigkeiten kausal relevant sind und Verhalten erwartbar machen, ist die gesellschaftstheoretische Präambel der Statistik und mit ihr weiter Teile der Soziologie. Sie ist der Grund dafür, weshalb Kategorien das Gerüst der amtlichen Statistik bilden und Individuen kategorial sortiert werden. Denn wenn Geschlecht, Beruf, ethnische Zugehörigkeit, Religion usw. nicht als folgenreich vorausgesetzt würden, müsste man sie auch nicht erheben. Statistiken legen mit anderen Worten eine Gesellschaft zugrunde, in der kategoriale Zugehörigkeiten stabil und der kategoriale Ort eines Individuums - die Schnittstelle seiner diversen kategorialen Zugehörigkeiten - folgenreich ist.

Recommendersysteme funktionieren anders und operieren unter anderen Prämissen. Sie stellen Vergleichbarkeit nicht über Kategorien her, sondern über die Vorgabe standardisierter Verhaltensoptionen (Abschn. 2.1). Sie setzen Kategorien nicht voraus, sondern erschließen kategoriale Zugehörigkeit aus dem Verhalten. Ihr Vorgehen folgt einem Bottom-up- und nicht wie die Statistik einem Top-down-Ansatz. Man ,ist“, was man hört, mag oder kauft, und was man ist, ist man immer nur temporär (Abschn. 2.2). Anstatt kategoriale Zugehörigkeiten als stabil zu behandeln, gehen Recommendersysteme davon aus, dass sie fluide sind und das Verhalten der bessere Prädiktor ist für zukünftiges Verhalten. Entsprechend sind sie vor allem an prädiktiven Analysen interessiert (Brayne 2021 in diesem Band sowie aus organisationssoziologischer Perspektive Büchner und Dosdall 2021 in diesem Band). Es geht nicht um die Frage, worin sich kategoriale Gruppen unterscheiden und weshalb, sondern darum, aus Verhaltensregelmäßigkeiten auf mögliches Verhalten in der Zukunft zu schließen (MacKenzie 2015). Da digitale Beobachtungsinstrumente keine kategorialen Vorannahmen treffen, gehen sie induktiv vor und behandeln Kategorien als flexible statistische Größen. Irgendwann, wenn man nur lange genug sammelt und rechnet, werden sich in den Daten Muster entdecken lassen, was auch immer

${ }^{21}$ Zur Humandifferenzierung als gesellschaftlichem Ordnungsprinzip vergleiche Hirschauer (2017) und aus systemtheoretischer Perspektive Nassehi (2017). 
deren Determinanten sind. Es braucht mit anderen Worten viele Daten - Big Data und viel Beobachtung - Big Observation -, um diesen Mustern auf die Spur zu kommen.

Mit der Verschiebung der Aufmerksamkeit von kategorialer Zugehörigkeit auf Verhaltensmuster geht auch eine veränderte Konzeption des Individuums einher. Die Bevölkerungsstatistik geht davon aus, dass Individuen durch stabile soziale Merkmale gekennzeichnet sind, von denen einige immer am Körper haften, andere erworben sind, aber sich nur langfristig ändern. Fisher und Mehozay (2019) sprechen in einem ähnlichen Zusammenhang von einer ,ascriptive conception of the individual“" und schreiben diese Auffassung einem Paradigma - einer ,scientific episteme“ - zu, die auch der Statistik zugrunde liegt: Individuen werden als Angehörige einer sozialen Kategorie modelliert unter Absehung ihrer individuellen Besonderheiten. Von dieser Auffassung unterscheiden sie eine ,,algorithmic episteme“, die von einem ganz anderen Begriff des Individuums ausgeht. Anstatt Individuen über ihre kategorialen Merkmale zu definieren, konzipieren sie sie in Termini ihrer Verhaltensmuster, die sich ständig ändern können. Die Frage, weshalb sich Individuen durch dieses oder jenes Verhalten auszeichnen, wird suspendiert. Es geht nicht mehr, um das Webersche Begriffspaar zu verwenden, um „Handeln“, sondern um „Verhalten“ - nicht um das Verstehen von Handlungen und deren Erklärung, sondern um die Identifikation von Verhaltensregelmäßigkeiten. Das zugrundeliegende Handlungsmodell ist ein radikal behavioristisches, das eng an die in der Verhaltensökonomie entwickelte Idee des Nudging gekoppelt ist, also an die Idee, durch geeignete Anreize ein gewünschtes Verhalten zu erzeugen (Cardon 2017; Seaver 2019). Nicht durch Zwang, sondern durch Verführung sollen Nutzer zu Käufen oder dazu gebracht werden, weiterhin Google zu konsultieren oder nur bei Netflix zu streamen. Dies alles vollzieht sich automatisch und weitgehend frei von Sinngebungsprozessen, im Unterschied zur Datenerhebung und -verarbeitung der Statistik, die trotz aller Standardisierung ein grundsätzlich soziales Unternehmen bleibt (Abschn. 3.2).

Recommendersysteme unterstellen mit anderen Worten eine Gesellschaft, die nicht mehr davon ausgeht, dass Individuen eine stabile Identität haben, die mit stabilen Meinungen und Präferenzen einhergeht. Ihre Elementareinheiten sind nicht Individuen, sondern die durch ihr Verhalten angezeigten Relationen zu Dingen oder andern Nutzern: „Users are known as a collection of relations to items and items are known as a collection of relations to users" (Seaver 2012). Dieses relationale Gefüge ändert sich mit jeder neuen Aktivität und in der Folge ändert sich auch die Identität des jeweiligen Nutzers: „Individual users are no more than the aggregation of the clicks they perform“ (Alaimo und Kallinikos 2017, S. 176). Die Konzeption des Sozialen als Netzwerk von Relationen macht die Unterscheidung zwischen Individual- und Sozialebene, auf der die amtliche Statistik und mit ihr ein Großteil der Soziologie gründet, hinfällig. Regelmäßigkeiten werden nicht aus aggregierten Individualdaten abgeleitet, sondern sie treten als temporäre Verdichtungen von Relationen, als Cluster, in Erscheinung. Was sich als Muster zeigt, wird nicht einer höheren Sozialebene zugeordnet - und schon gar nicht der „Gesellschaft“ -, sondern erscheint als momentanes Ordnungsmoment innerhalb eines ständigen Anbrandens von neuen Relationierungen (Latour et al. 2012). 
Dies bedeutet nicht, dass das Gesellschaftliche verschwindet und alles fluide wird. Ordnung - und damit Strukturen - gibt es nach wie vor, sonst könnten keine Regelmäßigkeiten entdeckt werden. Wenn das Verhalten der Nutzer nicht eine gewisse Konsistenz hätte, wären Amazon und Google nicht in der Lage, sie mit passenden Empfehlungen zu versorgen. Aber diese Konsistenz ist für digitale Beobachtungsinstrumente ein empirischer Sachverhalt, dessen Ursachen sie nicht notwendig ergründen müssen. Überspitzt könnte man sagen: Sie reagieren auf eine Gesellschaft, in der die Komplexität ein so hohes Maß angenommen hat, dass sie es aufgegeben hat, nach vereinheitlichenden Erklärungen zu suchen, und sich stattdessen das Soziale „operativ“ vorstellt, als einen ständigen Fluss von Verbindungen, die sich momenthaft zu Strukturen stabilisieren. Würde man in der Soziologie nach entsprechenden Sozialtheorien suchen, fielen einem am ehesten Theorien ein, die die Gesellschaft nicht mehr in Termini stabiler Strukturen begreifen, sondern sie aus einer relationalen und/oder prozessualen Perspektive beobachten. Beispiele dafür wären etwa die Systemtheorie, Andrew Abbotts prozessuale Soziologie oder Harrison Whites Variante der Netzwerktheorie.

Für Dominique Cardon (2017, S. 141) sind die digitalen Technologien ,eine Kampfansage an die lange statistische Tradition“ und das von ihr vorausgesetzte Gesellschaftsmodell. Der Nachweis eines Zusammenhanges zwischen Geschlecht und Erwerbstätigkeit oder Klassenlage und Wohnverhältnissen setzte eine Gesellschaft voraus, in der kategoriale Zugehörigkeiten weitgehend festlegten, welche Optionen dem Einzelnen offenstehen. Charles Tilly (1998) hat dafür den Begriff der „kategorialen Ungleichheit“ geprägt. Diese Voraussetzungen sind heute nicht mehr in gleichem Maße gegeben. Rogers Brubaker (2015) hat in diesem Zusammenhang und unter kritischer Bezugnahme auf Tilly eine zunehmende Entkopplung von „Differenz“ und „Ungleichheit“ diagnostiziert und gezeigt, dass zumindest im Falle von Geschlecht, Ethnie und Religion kategoriale Zugehörigkeiten nicht mehr in gleichem Maße als Platzanweiser fungieren wie noch in den 1960er- und 1970er-Jahren: „Inequality has become less categorical in recent decades, while categorical differences have become less inegalitarian“ (Brubaker 2015, S. 46; ausführlicher Heintz 2017). Gleichzeitig sind die kategorialen Grenzen durchlässiger geworden, indem sie vermehrt als kulturelle Grenzen interpretiert werden, die im Prinzip überschritten werden können (Brubaker 2016). Digitale Beobachtungsformate reagieren mit anderen Worten auf eine Gesellschaft, in der sich Individuen der Fixierung durch vorgegebene Kategorien zunehmend entziehen und sich die Determinanten ihres Verhaltens vervielfältigt haben. Für Cardon (2017) äußert sich diese Entwicklung in einer „Krise statistischer Konsistenz“: „Wenn die ,Gesellschaft der Berechnung“ dermaßen gründlich bis in die kleinsten Aspekte unser Leben vorgedrungen ist, dann deshalb, weil das Soziale nicht mehr die Konsistenz hat, die einst seine Darstellung und die Nutzung breiter und oberflächlicher Kategorien zur Beschreibung von Individuen erlaubte“ (Cardon 2017, S. 142; zu diesem Sachverhalt schon früh Beck 1983).

Dieser Wandel verweist auf eine Entwicklung, die Fourcade (2016) als „Ordinalisierung " bezeichnet, nämlich die Ablösung einer (nominalen) kategorialen Ordnung durch eine (ordinale) Ordnung, die von sozialen Merkmalen absieht und Individuen allein auf der Basis ihres Verhaltens bewertet. Anstatt Menschen kategorial zu unter- 
scheiden und sie auf dieser Basis in ein hierarchisches Verhältnis zu setzen, werden sie von ihren kategorialen Zuschreibungen befreit und als gewissermaßen „nackte“ Personen anhand identischer Bewertungsmaßstäbe verglichen und eingestuft. Ausdruck dieser zunehmenden Ordinalisierung ist etwa die Umstellung der Kriterien für die Kreditvergabe von kategorialen Merkmalen auf das individuelle Konsum- und Sparverhalten oder auch die Aufweichung der (nominalen) Unterscheidung von gesund und krank zugunsten eines graduellen Verständnisses von Gesundheit (Barnard und Fourcade 2021 in diesem Band). Das ist aber nur die eine Seite der Ordinalisierung und sie entspricht dem Selbstverständnis einer Gesellschaft, die sich als meritokratisch versteht und unter der Prämisse einer prinzipiellen Gleichheit aller operiert. Die andere Seite ist die, dass nominale Unterscheidungen unter der Hand wieder eingeführt werden und es am Ende wieder die bekannten kategorialen Gruppen sind, die als nicht kreditfähig eingestuft werden: Schwarze, Arbeitslose, alleinstehende Frauen mit Kindern (Fourcade und Healy 2013). Die Pointe bei Fourcade liegt darin, dass sie nicht bei der (modernisierungstheoretischen) Diagnose einer zunehmenden Versachlichung der Beurteilungskriterien und einer Abschwächung von Kollektivzugehörigkeiten stehenbleibt, sondern zeigt, dass kategoriale Ungleichheiten persistieren, aber anders hergestellt und gleichzeitig unsichtbar gemacht werden. Dies unterscheidet ihre Argumentation von Theorien wie etwa der Systemtheorie, die kategoriale Ungleichheiten als ,gesellschaftliche Residuen“ betrachtet, die der Operationslogik der Funktionssysteme entgegenstehen (Weinbach und Stichweh 2001, S. 35).

Digitale Technologien sind an der Erzeugung dieser widersprüchlichen Koexistenz von (sichtbarer) Ordinalität und (unsichtbarer) Nominalität aktiv beteiligt. Sie stellen die Algorithmen zur Verfügung, mit deren Hilfe Individuen ,gescort“ und anschließend wieder zu nominalen Gruppen ,,verklumpt“" werden. Aber sie sind nicht nur aktiv beteiligt, sondern bringen die Koexistenz dieser beiden Klassifikationsprinzipen auch in ihren eigenen Verfahrensweisen zum Ausdruck, indem sie z. B. Geschlecht oder Ethnizität nicht mehr als nominale Klassifikation behandeln, sondern „Frausein“ oder „Hispanicness“ auf einer kontinuierlichen Variable abbilden und erst im Nachhinein kategoriale Zuordnungen treffen (Fourcade und Healy 2017). So gesehen bereitet die Ordinalisierung der Gesellschaft (und die sie begleitende ReNominalisierung) den Boden für den Einsatz digitaler Technologien vor und wird durch diese gleichzeitig verstärkt. Der Ansatz von Fourcade lässt besser verstehen, weshalb das Beobachtungsformat der Statistik durch digitale Beobachtungsinstrumente Konkurrenz bekommen hat. Digitale Beobachtungstechnologien ersetzen die amtliche Statistik nicht, aber sie machen deutlich, dass es heute noch andere Weisen der Sozialbeobachtung gibt, die unter Umständen der gegenwärtigen Gesellschaft angepasster sind als die Beobachtung durch die Linsen der Sozialstatistik.

\footnotetext{
Danksagung Ich danke den Teilnehmerinnen und Teilnehmern des Autorenworkshops für die anregende Diskussion und insbesondere Hannah Bennani, Marion Müller, Martin Petzke, Tobias Werron und Markus Unternährer für ihr genaues Lesen und die hilfreichen Kommentare
}

Funding Open access funding provided by University of Luzern

Open Access Dieser Artikel wird unter der Creative Commons Namensnennung 4.0 International Lizenz veröffentlicht, welche die Nutzung, Vervielfältigung, Bearbeitung, Verbreitung und Wiedergabe in jeglichem Medium und Format erlaubt, sofern Sie den/die ursprünglichen Autor(en) und die Quelle ord- 
nungsgemäß nennen, einen Link zur Creative Commons Lizenz beifügen und angeben, ob Änderungen vorgenommen wurden.

Die in diesem Artikel enthaltenen Bilder und sonstiges Drittmaterial unterliegen ebenfalls der genannten Creative Commons Lizenz, sofern sich aus der Abbildungslegende nichts anderes ergibt. Sofern das betreffende Material nicht unter der genannten Creative Commons Lizenz steht und die betreffende Handlung nicht nach gesetzlichen Vorschriften erlaubt ist, ist für die oben aufgeführten Weiterverwendungen des Materials die Einwilligung des jeweiligen Rechteinhabers einzuholen.

Weitere Details zur Lizenz entnehmen Sie bitte der Lizenzinformation auf http://creativecommons.org/ licenses/by/4.0/deed.de.

\section{Literatur}

Agre, Philip E. 1994. Surveillance and capture: Two models of privacy. The Information Society 10:101-127.

Alaimo, Cristina, und Jannis Kallinikos. 2017. Computing the everyday: Social media as data platforms. The Information Society 33:175-191.

Alaimo, Cristina, und Jannis Kallinikos. 2019. Recommender System. In The Oxford Handbook of Media, Technology and Organization Studies, Hrsg. Timon Beyes, Robin Holt und Claus Pias, 400-411. Oxford: Oxford University Press.

Alaimo, Cristina, und Jannis Kallinikos. 2021. Managing by data: Algorithmic categories and organizing. Organization Studies, i. E.

Ambrose, Meg Leta. 2015. Lessons from the avalanche of numbers: Big Data in historical perspective. I/S: A Journal of Law and Policy for the Information Society 11.

Amoore, Louise, und Volha Piotukh. 2015. Life beyond big data: governing with little analytics. Economy and Society 44:341-366.

Arvidsson, Adam. 2004. On the "pre-history of the panoptic sort": Mobility in market research. Surveillance \& Society 1:456-474.

Baecker, Dirk. 2007. Studien zur nächsten Gesellschaft. Frankfurt a. M.: Suhrkamp.

Baecker, Dirk. 2018. 4.0 oder die Lücke die der Rechner lässt. Leipzig: Merve.

Bahrdt, Hans-Paul. 1958. Industriebürokratie. Stuttgart: Enke.

Barnard, Alex V., und Marion Fourcade. 2021. Das Unbehagen an der Ordinalisierung. In Soziale Praktiken des Beobachtens: Vergleichen, Bewerten, Kategorisieren und Quantifizieren, Kölner Zeitschrift für Soziologie und Sozialpsychologie Sonderheft 73, Hrsg. Bettina Heintz und Theresa Wobbe. Wiesbaden: Springer VS.

Beck, Ulrich. 1983. Jenseits von Klasse und Stand? Soziale Ungleichheit, gesellschaftliche Individualisierungstendenzen und die Entstehung neuer sozialer Formationen und Identitäten. Soziale Welt, Sonderband 2:35-74.

Beer, David. 2016. How should we do the history of Big Data. Big Data \& Society 2:1-10.

Behrisch, Lars. 2016. Die Berechnung der Glückseligkeit. Statistik und Politik in Deutschland und Frankreich im späten Ancien Régime. Ostfildern: Thorbecke.

Bennani, Hannah, und Marion Müller. 2021. „Who are we and how many?“ - Zur statistischen Konstruktion globaler Personenkategorien. In Soziale Praktiken des Beobachtens: Vergleichen, Bewerten, Kategorisieren und Quantifizieren, Kölner Zeitschrift für Soziologie und Sozialpsychologie Sonderheft 73, Hrsg. Bettina Heintz und Theresa Wobbe. Wiesbaden: Springer VS.

Biruk, Crystal. 2018. Cooking Data. Culture and Politics in an African Research World. Durham: Duke University Press.

Boltanski, Luc, und Laurent Thévenot. 1983. Finding one's way in social space: a study based on games. Social Science Information 22:631-680.

Bouk, Dan. 2015. How Our Days Became Numbered: Risk and the Rise of the Statistical Individual. Chicago: University of Chicago Press.

Brayne, Sarah. 2021. Überwachung durch Big Data - das Beispiel der Polizei. In Soziale Praktiken des Beobachtens: Vergleichen, Bewerten, Kategorisieren und Quantifizieren, Kölner Zeitschrift für Soziologie und Sozialpsychologie Sonderheft 73, Hrsg. Bettina Heintz und Theresa Wobbe. Wiesbaden: Springer VS.

Brubaker, Rogers. 2015. Grounds for Difference. Cambridge, Mass.: Harvard University Press. 
Brubaker, Rogers. 2016. Trans: Gender and Race in an Age of Unsettled Identities. Princeton: Princeton University Press.

Brückweh, Kerstin. 2015. Wissensproduktion durch britische Volkszählungen und Umfragen vom 19. Jahrhundert bis ins digitale Zeitalter. Berlin: deGruyter.

Büchner, Stefanie, und Henrik Dosdall. 2021. Organisation und Algorithmus. Wie algorithmische Kategorien, Vergleiche und Bewertungen durch Organisationen relevant gemacht werden. In Soziale Praktiken des Beobachtens: Vergleichen, Bewerten, Kategorisieren und Quantifizieren, Kölner Zeitschrift für Soziologie und Sozialpsychologie Sonderheft 73, Hrsg. Bettina Heintz und Theresa Wobbe. Wiesbaden: Springer VS.

Cainkar, Louise. 2006. The Social Construction of Difference and the Arab American Experience. Journal of American Ethnic History, 25:243-278.

Cakici, Baki, Evelyn Ruppert und Stephan Scheel. 2020. Peopling Europe through Data Practices. Science, Technology \& Human Values 45:199-211.

Cardon, Dominique. 2017. Den Algorithmus dekonstruieren. In Algorithmuskulturen, Hrsg. Robert Seyfert und Jonathan Roberge, 131-151. Bielefeld: transcript.

Cheney-Lippold, John. 2011. A New Algorithmic Identity. Soft Biopolitics and the Modulation of Control. Theory, Culture and Society 28:164-181.

Cheney-Lippold, John. 2017. We Are Data. Algorithms and the Making of Our Algorithmical Selves. New York: New York University Press.

Cicourel, Aaron V. 1970. Methode und Messung in der Soziologie. Frankfurt a. M.: Suhrkamp.

Degli-Espositi, Sara. 2014. When big data meets dataveillance: The hidden side of analytics. Surveillance \& Society 12:209-225.

Desrosières, Alain. 2005. Die Politik der großen Zahlen. Eine Geschichte der statistischen Denkweise. Heidelberg: Springer.

Desrosières, Alain. 2010. Pour une sociologie historique de la quantification. L'argument statistique I. Paris: Presses des Mines.

Desrosières, Alain. 2015. Retroaction: How Indicators Feed Back Onto Quantified Actors. In The World of Indicators. The Making of Governmental Knowledge through Quantification, Hrsg. Richard Rottenburg, 329-353. Cambridge: Cambridge University Press.

Diaz-Bone, Rainer. 2018. Die „Economie des Conventions“. Grundlagen und Entwicklungen der französischen Wirtschaftssoziologe. Wiesbaden: Springer VS.

Durkheim, Emile. 1983/1897. Der Selbstmord. Frankfurt a.M.: Suhrkamp.

Durkheim, Emile. 1984/1895. Die Regeln der soziologischen Methode. Frankfurt a. M.: Suhrkamp.

Fisher, Eran, und Yoav Mehozay. 2019. How algorithms see their audience: media epistemes and the changing conception of the individual. Media, Culture \& Society 41:1179-1191.

Foucault, Michel. 2001. In Verteidigung der Gesellschaft. Frankfurt a. M.: Suhrkamp.

Foucault, Michel. 2004. Sicherheit, Territorium, Bevölkerung. Geschichte der Gouvernementalität I. Frankfurt a. M.: Suhrkamp.

Fourcade, Marion. 2016. Ordinalization. Lewis A. Coser Memorial Award for Theoretical Agenda Setting 2014. Sociological Theory 34: 175-195.

Fourcade, Marion, und Kieran Healy. 2013. Classification Situations: Life-chances in the Neoliberal Era. Accounting, Organizations, and Society 38:559-72.

Fourcade, Marion, und Kieran Healy. 2017. Categories All the Way Down. Historical Social Research 42:286-296.

Fourcade, Marion, und Daniel N. Kluttz. 2020. A Maussian bargain. Accumulation by gift in the digital economy. Big Data \& Society 7:1-16.

Garfinkel, Harold. 1967. Studies in Ethnomethodology. Englewood Cliffs, NJ: Prentice Hall.

Gerlitz, Carolin. 2016. What Counts? Reflections on the Multivalence of Social Media Data. Digital Culture and Society 2:19-38.

Gitelman, Lisa. 2013. "Raw Data" is an Oxymoron. Cambridge: MIT Press.

Hacking, Ian.1982. Biopower and the Avalanche of Printed Numbers. Humanities and Society 5:279-295.

Hacking, Ian. 1986. Making up people. In Reconstructing individualism. Autonomy, individuality, and the self in Western thought, Hrsg. Thomas C. Heller und Christine Brooke-Rose, 222-237. Stanford: Stanford University Press.

Hacking, Ian. 1994. How Numerical Sociology Began by Counting Suicides: From Medical Pathology to Social Pathology. In The Natural Sciences and the Social Sciences, Hrsg. Bernard I. Cohen, 101-133. Dordrecht: Kluwer.

Haggerty, Kevin D., und Richard V. Ericson. 2000. The surveillant assemblage. British Journal of Socio$\log y$ 51:605-622. 
Heintz, Bettina. 1990. Das Fließband im Kopf. Computer und Rationalisierung. In Schweiz im Wandel. Studien zur neueren Gesellschaftsgeschichte, Hrsg. Sebastian Brändli, David Gugerli, Rudolf Jaun und Ulrich Pfister, 117-147. Basel: Helbling und Lichtenhahn.

Heintz, Bettina. 1993. Die Herrschaft der Regel. Zur Grundlagengeschichte des Computers. Frankfurt a. M.: Campus.

Heintz, Bettina. 2010. Numerische Differenz. Überlegungen zu einer Soziologie des (quantitativen) Vergleichs. Zeitschrift für Soziologie 39:162-181.

Heintz, Bettina. 2012. Welterzeugung durch Zahlen Modelle politischer Differenzierung in internationalen Statistiken, 1948-2010. In Welterzeugung durch Bilder, Hrsg. Cornelia Bohn, Arno Schubach und Leo Wansleben, 7-39. Stuttgart: Lucius \& Lucius.

Heintz, Bettina. 2017. Kategoriale Ungleichheit und die Anerkennung von Differenz. In Un/doing differences. Praktiken der Humandifferenzierung, Hrsg. Stefan Hirschauer, 79-115. Weilerswist: Velbrück.

Hirschauer, Stefan. 2017. Humandifferenzierung. Modi und Grade sozialer Zugehörigkeit. In Un/doing differences. Praktiken der Humandifferenzierung, Hrsg. Stefan Hirschauer, 29-54. Weilerswist: Velbrück.

Igo, Sarah E. 2007. The Averaged American: Surveys, Citizens, and the Making of a Mass Public. Cambridge, Mass.: Harvard University Press.

Igo, Sarah E. 2018. The Known Citizen. A History of Privacy in Modern America. Cambridge, Mass.: Harvard University Press.

Jerven, Morten. 2013. Poor Numbers: How We Are Misled by African Development Statistics and What to Do About It. Ithaca, NY: Cornell University Press.

Keller, Felix. 2001. Archäologie der Meinungsforschung. Konstanz: UVK.

Knorr Cetina, Karin. 1984. Die Fabrikation von Erkenntnis. Frankfurt a. M.: Suhrkamp.

Köhler, Benedikt. 2008. Amtliche Statistik, Sichtbarkeit und die Herstellung von Verfügbarkeit. Berliner Journal für Soziologie 18:73-98.

Krohn, Wolfgang, und Johannes Weyer. 1989. Gesellschaft als Labor. Soziale Welt 40:349-373.

Latour, Bruno, und Steve Woolgar. 1979. Laboratory Life: The Social Construction of Scientific Facts. Princeton: Princeton University Press.

Latour, Bruno, Pablo Jensen et al. 2012. "The whole is always smaller than its parts"- a digital test of Gabriel Tarde's Monad. British Journal of Sociology 63:590-615.

Link, Jürgen. 2009. Versuch über den Normalismus. Wie Normalität produziert wird. Göttingen: Vandenhoeck \& Ruprecht.

Lury, Celia, und Sophie Day. 2019. Algorithmic Personalization as a Mode of Individuation. Theory, Culture \& Society 36:17-37.

MacKenzie, Adrian. 2015. The production of prediction: What does machine learning want? European Journal of Cultural Studies 18:429-445.

Mair, Michael, und Christian Greiffenhagen. 2016. Statistical Practice: Putting Society on Display. Theory, Culture \& Society 33: 51-77.

Maynard, Douglas W., und Nora Kate Schaeffer. 2000. Toward a Sociology of Social Scientific Knowledge: Survey Research and Ethnomethodology's Asymmetric Alternates. Social Studies of Science 30:323-370.

Mennicken, Andrea, und Martin Kornberger. 2021. Von Performativität zu Generativität. Bewertung und ihre Folgen im Kontext der Digitalisierung. In Soziale Praktiken des Beobachtens: Vergleichen, Bewerten, Kategorisieren und Quantifizieren, Kölner Zeitschrift für Soziologie und Sozialpsychologie Sonderheft 73, Hrsg. Bettina Heintz und Theresa Wobbe. Wiesbaden: Springer VS.

Nassehi, Armin. 2017. Humandifferenzierung und gesellschaftliche Differenzierung. Eine Verhältnisbestimmung. In Un/doing differences. Praktiken der Humandifferenzierung, Hrsg. Stefan Hirschauer, 55-78. Weilerswist: Velbrück.

Nassehi, Armin. 2019. Muster. Theorie der digitalen Gesellschaft. Frankfurt a. M.: Suhrkamp.

Paßmann, Johannes, und Carolin Gerlitz. 2014. "Good" Platform-Political Reasons for "Bad" PlatformData. Zur sozio-Technischen Geschichte der Plattformaktivitäten Fav, Retweet und Like. Mediale Kontrolle unter Beobachtung 3:1-40.

Passoth, Jan-Hendrik, und Werner Rammert. 2020. Digitale Technik entspricht digitaler Gesellschaft? Soziologische Revue 43:312-320.

Penissat, Etienne, Cécile Brousse et al. 2016. From statistical categorizations to ordinary categorizations of social space. History and legacy of an original study based on a card game. Historical Social Research 41:135-154. 
Petzke, Martin. 2021. Die Moral des Vermessens. Bewertungsüberschüsse in der statistischen Beobachtung von Integrationsfortschritten der Migrantenbevölkerung in Deutschland. In Soziale Praktiken des Beobachtens: Vergleichen, Bewerten, Kategorisieren und Quantifizieren, Kölner Zeitschrift für Soziologie und Sozialpsychologie Sonderheft 73, Hrsg. Bettina Heintz und Theresa Wobbe. Wiesbaden: Springer VS.

Porter, Theodore W. 1986. The Rise of Statistical Thinking. 1820-1900. Princeton: Princeton University Press.

Porter, Theodore W. 1992. Quantification and the Accounting Ideal in Science. Social Studies of Science 22:633-651.

Porter, Theodore W. 1995. Trust in Numbers. The Pursuit of Objectivity in Science and Public Life. Princeton: Princeton University Press.

Prey, Robert. 2018. Nothing personal: algorithmic individuation on music streaming platforms. Media, Culture \& Society 40:1086-1100.

Quantcast. 2014. Understanding Digital Audience Measurement. www.quantcast.com

Quetelet, Adolphe.1838. Über den Menschen und die Entwicklung seiner Fähigkeiten, oder Versuch einer Physik der Gesellschaft. Stuttgart: E. Schweizerbart's Verlagshandlung.

Reckwitz, Andreas. 2017. Die Gesellschaft der Singularitäten. Zum Strukturwandel der Moderne. Frankfurt a. M.: Suhrkamp.

Renard, Léa. 2021. Vergleichsverbot? Bevölkerungsstatistiken und die Frage der Vergleichbarkeit in den deutschen Kolonien (1885-1914). In Soziale Praktiken des Beobachtens: Vergleichen, Bewerten, Kategorisieren und Quantifizieren, Kölner Zeitschrift für Soziologie und Sozialpsychologie Sonderheft 73, Hrsg. Bettina Heintz und Theresa Wobbe. Wiesbaden: Springer VS.

Ruppert, Evelyn. 2009a. Becoming Peoples: "Counting Heads in Northern Wilds". Journal of Cultural Economy 2:11-31.

Ruppert, Evelyn. 2009b. "I Is; Therefore I Am": The Census as Practice of Double Identification. Sociological Research Online 13.

Ruppert, Evelyn. 2011. Population Objects: Interpassive Subjects. Sociology 45: 218-233.

Schneider, Michael C. 2013. Wissensproduktion im Staat. Das königlich preußische statistische Bureau, 1860-1914. Frankfurt a. M.: Campus.

Scott, James. 1999. Seeing Like a State: How Certain Schemes to Improve the Human Condition Have Failed. New Haven: Yale University Press.

Seaver, Nick. 2019. Captivating algorithms: Recommender systems as traps. Journal of Material Culture 24:421-436.

Seaver, Nick. 2012. Algorithmic recommendations and synaptic functions. Limn 2. http://limn.it/ algorithmic-recommendations-and-synaptic-functions.

Segelken, Barbara. 2010. Bilder des Staates. Kammer, Kasten und Tafel als Visualisierungen staatlicher Zusammenhänge. Berlin: Akademie Verlag.

Smith, Brent, und Greg Linden. 2017. Two Decades of Amazon Recommendations at Amazon.com. IEEE Internet Computing:12-18.

Steinmetz, Willibald. 2021. Macht - Leistung - Kultur: Staatenvergleiche vom 17. bis ins frühe 20. Jahrhundert. In Soziale Praktiken des Beobachtens: Vergleichen, Bewerten, Kategorisieren und Quantifizieren, Kölner Zeitschrift für Soziologie und Sozialpsychologie Sonderheft 73, Hrsg. Bettina Heintz und Theresa Wobbe. Wiesbaden: Springer VS.

Stevens, S. S. 1946. On the Theory of Scales of Measurements. Science 103:677-680.

Suchman, Lucy, und Brigitte Jordan. 1990. Interactional Troubles in Face-to-Face Survey Interviews. Journal of the American Statistical Association 85:232-241.

Supik, Linda, und Riem Spielhaus. 2019. Introduction to Special Issue: Matters of classification and representation: Quantifying ethnicity, religion and migration. Ethnicities 19:455-468.

Tenbruck, Friedrich H. 1990. Emile Durkheim oder die Geburt der Gesellschaft aus dem Geist der Soziologie. In Die kulturellen Grundlagen der Gesellschaft, Hrsg. Friedrich H. Tenbruck, 198-211. Opladen: Westdeutscher Verlag.

Thévenot, Laurent. 2016. From Social Coding to Economics of Convention: A Thirty-Year Perspective on the Analysis of Qualification and Quantification Investments. Historical Social Research 41:96-117.

Tilly, Charles. 1998. Durable Inequality. Berkeley: University of California Press.

Turow, Joseph.1997. Breaking up America. Advertising and the new media world. Chicago: Chicago University Press.

Unternährer, Markus. 2020. „User item item user“. Zur Vergleichspraxis von Recommendersystemen. In Global beobachten und vergleichen. Soziologische Analysen zur Weltgesellschaft, Hrsg. Hannah Bennani, Martin Bühler, Sophia Cramer und Andrea Glauser, 381-408. Frankfurt a. M.: Campus. 
Unternährer, Markus. 2021a. Die Ordnung der Empfehlung. In Soziale Praktiken des Beobachtens: Vergleichen, Bewerten, Kategorisieren und Quantifizieren, Kölner Zeitschrift für Soziologie und Sozialpsychologie Sonderheft 73, Hrsg. Bettina Heintz und Theresa Wobbe. Wiesbaden: Springer VS.

Unternährer, Markus. 2021b. Momente der Datafizierung. Dissertation, Universität Luzern.

Von Oertzen, Christine. 2017a. Die Historizität der Verdatung: Konzepte, Werkzeuge und Praktiken im 19. Jahrhundert. NTM Zeitschrift für Geschichte der Wissenschaften, Technik und Medizin 25:407-434.

Von Oertzen, Christine. 2017b. Machineries of Data Power: Manual versus Mechanical Census Compilation in Nineteenth Century Europe. Osiris 32:129-150.

Weinbach, Christine, und Rudolf Stichweh. 2001. Die Geschlechterdifferenz in der funktional differenzierten Gesellschaft. In Geschlechtersoziologie. Sonderband 41 der Kölner Zeitschrift für Soziologie und Sozialpsychologie, Hrsg. Bettina Heintz, 30-52. Wiesbaden: Westdeutscher Verlag.

Wobbe, Theresa. 2021. Die Differenz Haushalt vs. Markt als latentes Beobachtungsschema. Vergleichsverfahren der inter/nationalen Statistik (1882-1990). In Soziale Praktiken des Beobachtens: Vergleichen, Bewerten, Kategorisieren und Quantifizieren, Kölner Zeitschrift für Soziologie und Sozialpsychologie Sonderheft 73, Hrsg. Bettina Heintz und Theresa Wobbe. Wiesbaden: Springer VS.

Yeung, Karen. 2016. "Hypernudge". Big Data as a mode of regulation by design. Information, Communication \& Society 20:118-136.

Zerubavel, Eviatar. 1996. Lumping and splitting: Notes on social classification. Sociological Forum 11:421-433.

Zerubavel, Eviatar. 2018. Taken for Granted. The Remarkable Power of the Unremarkable. Princeton: Princeton University Press.

Bettina Heintz 1949, Dr. phil., Sen. Prof. für Soziologie, Universität Luzern. Forschungsgebiete: Soziologie des Vergleichs und der Quantifizierung, Soziologie der Weltgesellschaft, Menschenrechte, soziologische Theorie. Veröffentlichungen: „Wir leben im Zeitalter der Vergleichung.“ Perspektiven einer Soziologie des Vergleichs, Zeitschrift für Soziologie 45, 2016; Good - Better - Best: Comparisons and the Power of Ranking Orders, in: W. Steinmetz (Hrsg.), The Force of Comparison: A New Perspective on Modern European History and the Contemporary World. New York 2019; Menschenrechte in der Weltgesellschaft. Deutungswandel und Wirkungsweise eines globalen Leitwertes. Frankfurt a. M. (als Hrsg., mit B. Leisering). 